\title{
Inherent Limitations of Hybrid Transactional Memory
}

\author{
Dan Alistarh $^{1} \quad$ Justin Kopinsky $^{4}$ Petr Kuznetsov ${ }^{2} \quad$ Srivatsan Ravi $^{3} \quad$ Nir Shavit $^{4,5}$ \\ ${ }^{1}$ Microsoft Research, Cambridge \\ ${ }^{2}$ Télécom ParisTech \\ ${ }^{3} \mathrm{TU}$ Berlin \\ ${ }^{4}$ Massachusetts Institute of Technology \\ ${ }^{5}$ Tel Aviv University
}

\begin{abstract}
Several Hybrid Transactional Memory (HyTM) schemes have recently been proposed to complement the fast, but best-effort nature of Hardware Transactional Memory (HTM) with a slow, reliable software backup. However, the costs of providing concurrency between hardware and software transactions in HyTM are still not well understood.

In this paper, we propose a general model for HyTM implementations, which captures the ability of hardware transactions to buffer memory accesses. The model allows us to formally quantify and analyze the amount of overhead (instrumentation) caused by the potential presence of software transactions. We prove that (1) it is impossible to build a strictly serializable HyTM implementation that has both uninstrumented reads and writes, even for very weak progress guarantees, and (2) the instrumentation cost incurred by a hardware transaction in any progressive opaque HyTM may get linear in the transaction's data set. We further describe two implementations that, for two different progress conditions, exhibit optimal instrumentation costs. In sum, this paper captures for the first time an inherent trade-off between the degree of hardware-software TM concurrency and the amount of incurred instrumentation overhead.
\end{abstract}




\section{Introduction}

Hybrid transactional memory. Ever since its introduction by Herlihy and Moss [24], Transactional Memory (TM) has promised to be an extremely useful tool, with the power to fundamentally change concurrent programming. It is therefore not surprising that the recently introduced Hardware Transactional Memory (HTM) implementations [1, 30, 31] have been eagerly anticipated and scrutinized by the community.

Early experience with programming HTM, e.g. [3, 12, 14, paints an interesting picture: if used carefully, HTM can be an extremely useful construct, and can significantly speed up and simplify concurrent implementations. At the same time, this powerful tool is not without its limitations: since HTMs are usually implemented on top of the cache coherence mechanism, hardware transactions have inherent capacity constraints on the number of distinct memory locations that can be accessed inside a single transaction. Moreover, all current proposals are best-effort, as they may abort under imprecisely specified conditions. In brief, the programmer should not solely rely on HTMs.

Several Hybrid Transactional Memory (HyTM) schemes [9, 11,26,28 have been proposed to complement the fast, but best-effort nature of HTM with a slow, reliable software transactional memory (STM) backup. These proposals have explored a wide range of trade-offs between the overhead on hardware transactions, concurrent execution of hardware and software, and the provided progress guarantees.

Early proposals for HyTM implementations [11,26] shared some interesting features. First, transactions that do not conflict are expected to run concurrently, regardless of their types (software or hardware). This property is referred to as progressiveness [19] and is believed to allow for increased parallelism. Second, in addition to exchanging the values of transactional objects, hardware transactions usually employ code instrumentation techniques. Intuitively, instrumentation is used by hardware transactions to detect concurrency scenarios and abort in the case of contention. The number of instrumentation steps performed by these implementations within a hardware transaction is usually proportional to the size of the transaction's data set.

Recent work by Riegel et al. [33] surveyed the various HyTM algorithms to date, focusing on techniques to reduce instrumentation overheads in the frequently executed hardware fast-path. However, it is not clear whether there are fundamental limitations when building a HyTM with non-trivial concurrency between hardware and software transactions. In particular, what are the inherent instrumentation costs of building a HyTM, and what are the trade-offs between these costs and the provided concurrency, i.e., the ability of the HyTM system to run software and hardware transactions in parallel?

Modelling HyTM. To address these questions, we propose the first model for hybrid TM systems which formally captures the notion of cached accesses provided by hardware transactions, and precisely defines instrumentation costs in a quantifiable way.

We model a hardware transaction as a series of memory accesses that operate on locally cached copies of the variables, followed by a cache-commit operation. In case a concurrent transaction performs a (read-write or write-write) conflicting access to a cached object, the cached copy is invalidated and the hardware transaction aborts.

Our model for instrumentation is motivated by recent experimental evidence which suggests that the overhead on hardware transactions imposed by code which detects concurrent software transactions is a significant performance bottleneck [29]. In particular, we say that a HyTM implementation imposes a logical partitioning of shared memory into data and metadata locations. Intuitively, metadata is used by transactions to exchange information about contention and conflicts while data locations only store the values of data items read and updated within transactions. We quantify instrumentation cost by measuring the number of accesses to metadata objects which transactions perform.

The cost of instrumentation. Once this general model is in place, we derive two lower 
bounds on the cost of implementing a HyTM. First, we show that some instrumentation is necessary in a HyTM implementation even if we only intend to provide sequential progress, where a transaction is only guaranteed to commit if it runs in the absence of concurrency.

Second, we prove that any progressive HyTM implementation providing obstruction-free liveness (every operation running solo returns some response) and has executions in which an arbitrarily long read-only hardware transaction running in the absence of concurrency must access a number of distinct metadata objects proportional to the size of its data set. We match this lower bound with an HyTM algorithm that, additionally, allows for uninstrumented writes and invisible reads.

Low-instrumentation HyTM. The high instrumentation costs of early HyTM designs, which we show to be inherent, stimulated more recent HyTM schemes $9,28,29,33$ to sacrifice progressiveness for constant instrumentation cost (i.e., not depending on the size of the transaction). In the past two years, Dalessandro et al. [9] and Riegel et al. [33 have proposed HyTMs based on the efficient NOrec STM [10]. These HyTMs schemes do not guarantee any parallelism among transactions; only sequential progress is ensured. Despite this, they are among the best-performing HyTMs to date due to the limited instrumentation in hardware transactions.

Starting from this observation, we provide a more precise upper bound for low-instrumentation HyTMs by presenting a HyTM algorithm with invisible reads and uninstrumented hardware writes which guarantees that a hardware transaction accesses at most one metadata object in the course of its execution. Software transactions in this implementation remain progressive, while hardware transactions are guaranteed to commit only if they do not run concurrently with an updating software transaction (or exceed capacity). Therefore, the cost of avoiding the linear lower bound for progressive implementations is that hardware transactions may be aborted by non-conflicting software ones.

In sum, this paper captures for the first time an inherent trade-off between the degree of concurrency between hardware and software transactions provided a HyTM implementation and the incurred amount of instrumentation overhead.

Roadmap. The rest of the paper is organized as follows. Section 2 introduces the basic TM model and definitions. Section 3 presents our model of HyTM implementations, and Section 4 formally defines instrumentation. Sections 5 proves the impossibility of implementing uninstrumented HyTMs, while Section 6 establishes a linear tight bound on metadata accesses for progressive HyTMs. Section 7 describes an algorithm that overcomes this linear cost by weakening progress. Section 8 presents the related work and Section 9 concludes the paper. The Appendix contains the pseudo-code of the algorithms presented in this paper and their proofs of correctness.

\section{Preliminaries}

Transactional Memory (TM). A transaction is a sequence of transactional operations (or t-operations), reads and writes, performed on a set of transactional objects (t-objects). A transactional memory implementation provides a set of concurrent processes with deterministic algorithms that implement reads and writes on t-objects using a set of base objects.

More precisely, for each transaction $T_{k}$, a TM implementation must support the following t-operations: $\operatorname{read}_{k}(X)$, where $X$ is a t-object, that returns a value in a domain $V$ or a special value $A_{k} \notin V$ (abort), write $(X, v)$, for a value $v \in V$, that returns ok or $A_{k}$, and $\operatorname{try} C_{k}$ that returns $C_{k} \notin V$ (commit) or $A_{k}$.

Configurations and executions. A configuration of a TM implementation specifies the state of each base object and each process. In the initial configuration, each base object has its initial value and each process is in its initial state. An event (or step) of a transaction invoked by some process is an invocation of a t-operation, a response of a t-operation, or an atomic primitive 
operation applied to base object along with its response. An execution fragment is a (finite or infinite) sequence of events $E=e_{1}, e_{2}, \ldots$ An execution of a TM implementation $\mathcal{M}$ is an execution fragment where, informally, each event respects the specification of base objects and the algorithms specified by $\mathcal{M}$. In the next section, we define precisely how base objects should behave in a hybrid model combining direct memory accesses with cached accesses (hardware transactions).

The read set (resp., the write set) of a transaction $T_{k}$ in an execution $E$, $\operatorname{denoted} \operatorname{Rset}_{E}\left(T_{k}\right)$ (and resp. $W_{s e t}\left(T_{k}\right)$ ), is the set of t-objects that $T_{k}$ attempts to read (and resp. write) by issuing a t-read (and resp. t-write) invocation in $E$ (for brevity, we sometimes omit the subscript $E$ from the notation). The data set of $T_{k}$ is $\operatorname{Dset}\left(T_{k}\right)=\operatorname{Rset}\left(T_{k}\right) \cup \operatorname{Wset}\left(T_{k}\right) . T_{k}$ is called read-only if $W \operatorname{set}\left(T_{k}\right)=\emptyset$; write-only if $\operatorname{Rset}\left(T_{k}\right)=\emptyset$ and updating if $W \operatorname{set}\left(T_{k}\right) \neq \emptyset$. Note that we consider the conventional dynamic TM model: the data set of a transaction is not known apriori (i.e., at the start of the transaction) and it is identifiable only by the set of t-objects the transaction has invoked a read or write in the given execution.

For any finite execution $E$ and execution fragment $E^{\prime}, E \cdot E^{\prime}$ denotes the concatenation of $E$ and $E^{\prime}$ and we say that $E \cdot E^{\prime}$ is an extension of $E$. For every transaction identifier $k, E \mid k$ denotes the subsequence of $E$ restricted to events of transaction $T_{k}$. If $E \mid k$ is non-empty, we say that $T_{k}$ participates in $E$, and let $\operatorname{txns}(E)$ denote the set of transactions that participate in $E$. Two executions $E$ and $E^{\prime}$ are indistinguishable to a set $\mathcal{T}$ of transactions, if for each transaction $T_{k} \in \mathcal{T}, E\left|k=E^{\prime}\right| k$.

Complete and incomplete transactions. A transaction $T_{k} \in \operatorname{txns}(E)$ is complete in $E$ if $E \mid k$ ends with a response event. The execution $E$ is complete if all transactions in $\operatorname{txns}(E)$ are complete in $E$. A transaction $T_{k} \in \operatorname{txns}(E)$ is $t$-complete if $E \mid k$ ends with $A_{k}$ or $C_{k}$; otherwise, $T_{k}$ is t-incomplete. $T_{k}$ is committed (resp. aborted) in $E$ if the last event of $T_{k}$ is $C_{k}$ (resp. $A_{k}$ ). The execution $E$ is t-complete if all transactions in $\operatorname{txns}(E)$ are t-complete. A configuration $C$ after an execution $E$ is quiescent (resp. t-quiescent) if every transaction $T_{k} \in \operatorname{txns}(E)$ is complete (resp. t-complete) in $E$.

Contention. We assume that base objects are accessed with read-modify-write ( $\mathrm{rmw}$ ) primitives [15, 22]. A rmw primitive $\langle g, h\rangle$ applied to a base object atomically updates the value of the object with a new value, which is a function $g(v)$ of the old value $v$, and returns a response $h(v)$. A rmw primitive event on a base object is trivial if, in any configuration, its application does not change the state of the object. Otherwise, it is called nontrivial.

Events $e$ and $e^{\prime}$ of an execution $E$ contend on a base object $b$ if they are both primitives on $b$ in $E$ and at least one of them is nontrivial.

In a configuration $C$ after an execution $E$, every incomplete transaction $T$ has exactly one enabled event in $C$, which is the next event $T$ will perform according to the TM implementation.

We say that a transaction $T$ is poised to apply an event $e$ after $E$ if $e$ is the next enabled event for $T$ in $E$. We say that transactions $T$ and $T^{\prime}$ concurrently contend on $b$ in $E$ if they are each poised to apply contending events on $b$ after $E$.

We say that an execution fragment $E$ is step contention-free for t-operation $o p_{k}$ if the events of $E \mid o p_{k}$ are contiguous in $E$. An execution fragment $E$ is step contention-free for $T_{k}$ if the events of $E \mid k$ are contiguous in $E$, and $E$ is step contention-free if $E$ is step contention-free for all transactions that participate in $E$.

TM correctness. A history $H$ exported by an execution fragment $E$, denoted $H_{E}$, is the subsequence of $E$ consisting of only the invocation and response events of t-operations. Two histories $H$ and $H^{\prime}$ are equivalent if $\operatorname{txns}(H)=\operatorname{txns}\left(H^{\prime}\right)$ and for every transaction $T_{k} \in \operatorname{txns}(H)$, $H\left|k=H^{\prime}\right| k$. We say that two execution fragments $E$ and $E^{\prime}$ are similar if $H$ and $H^{\prime}$ are equivalent, where $H$ (and resp. $H^{\prime}$ ) is the history exported by $E$ (and resp. $E^{\prime}$ ). For any two transactions $T_{k}, T_{m} \in \operatorname{txns}(E)$, we say that $T_{k}$ precedes $T_{m}$ in the real-time order of $E$ $\left(T_{k} \prec_{E}^{R T} T_{m}\right)$ if $T_{k}$ is t-complete in $E$ and the last event of $T_{k}$ precedes the first event of $T_{m}$ in $E$. If neither $T_{k}$ precedes $T_{m}$ nor $T_{m}$ precedes $T_{k}$ in real-time order, then $T_{k}$ and $T_{m}$ are 
concurrent in $E$. An execution $E$ is sequential if every invocation of a t-operation is either the last event in $H$ or is immediately followed by a matching response, where $H$ is the history exported by $E$. An execution $E$ is $t$-sequential if there are no concurrent transactions in $E$.

We say that $\operatorname{read}_{k}(X)$ is legal in a t-sequential execution $E$ if it returns the latest written value of $X$, and $E$ is legal if every $\operatorname{read}_{k}(X)$ in $H$ that does not return $A_{k}$ is legal in $E$. Informally, a history $H$ is opaque if there exists a legal t-sequential history $S$ equivalent to $H$ that respects the real-time order of transactions in $H$ [20]. A weaker condition called strict serializability ensures opacity only with respect to committed transactions. Formal definitions are delegated to Appendix A.

TM-liveness. A liveness property specifies the conditions under which a t-operation must return. A TM implementation provides wait-free (WF) TM-liveness if it ensures that every toperation returns in a finite number of its steps. A weaker property of obstruction-freedom (OF) ensures that every operation running step contention-free returns in a finite number of its own steps. The weakest property we consider here is sequential TM-liveness that only guarantees that t-operations running in the absence of concurrent transactions returns in a finite number of its steps.

\section{Hybrid Transactional Memory (HyTM)}

Direct accesses and cached accesses. We now describe the operation of a Hybrid Transactional Memory (HyTM) implementation. In our model, every base object can be accessed with two kinds of primitives, direct and cached.

In a direct access, the rmw primitive operates on the memory state: the direct-access event atomically reads the value of the object in the shared memory and, if necessary, modifies it.

In a cached access performed by a process $i$, the rmw primitive operates on the cached state recorded in process $i$ 's tracking set $\tau_{i}$. One can think of $\tau_{i}$ as the $L 1$ cache of process $i$. A a hardware transaction is a series of cached rmw primitives performed on $\tau_{i}$ followed by a cache-commit primitive.

More precisely, $\tau_{i}$ is a set of triples $(b, v, m)$ where $b$ is a base object identifier, $v$ is a value, and $m \in\{$ shared, exclusive $\}$ is an access mode. The triple $(b, v, m)$ is added to the tracking set when $i$ performs a cached rmw access of $b$, where $m$ is set to exclusive if the access is nontrivial, and to shared otherwise. We assume that there exists some constant TS (representing the size of the L1 cache) such that the condition $\left|\tau_{i}\right| \leq T S$ must always hold; this condition will be enforced by our model. A base object $b$ is present in $\tau_{i}$ with mode $m$ if $\exists v,(b, v, m) \in \tau_{i}$.

A trivial (resp. nontrivial) cached primitive $\langle g, h\rangle$ applied to $b$ by process $i$ first checks the condition $\left|\tau_{i}\right|=T S$ and if so, it sets $\tau_{i}=\emptyset$ and immediately returns $\perp$ (we call this event a capacity abort). We assume that $T S$ is large enough so that no transaction with data set of size 1 can incur a capacity abort. If the transaction does not incur a capacity abort, the process checks whether $b$ is present in exclusive (resp. any) mode in $\tau_{j}$ for any $j \neq i$. If so, $\tau_{i}$ is set to $\emptyset$ and the primitive returns $\perp$. Otherwise, the triple $(b, v$, shared $)$ (resp. $(b, g(v)$, exclusive $)$ ) is added to $\tau_{i}$, where $v$ is the most recent cached value of $b$ in $\tau_{i}$ (in case $b$ was previously accessed by $i$ within the current hardware transaction) or the value of $b$ in the current memory configuration, and finally $h(v)$ is returned.

A tracking set can be invalidated by a concurrent process: if, in a configuration $C$ where $(b, v$, exclusive $) \in \tau_{i}$ (resp. $(b, v$, shared $\left.) \in \tau_{i}\right)$, a process $j \neq i$ applies any primitive (resp. any nontrivial primitive) to $b$, then $\tau_{i}$ becomes invalid and any subsequent cached primitive invoked by $i$ sets $\tau_{i}$ to $\emptyset$ and returns $\perp$. We refer to this event as a tracking set abort.

Finally, the cache-commit primitive issued by process $i$ with a valid $\tau_{i}$ does the following: for each base object $b$ such that $(b, v$, exclusive $) \in \tau_{i}$, the value of $b$ in $C$ is updated to $v$. Finally, $\tau_{i}$ is set to $\emptyset$ and the primitive returns commit. 


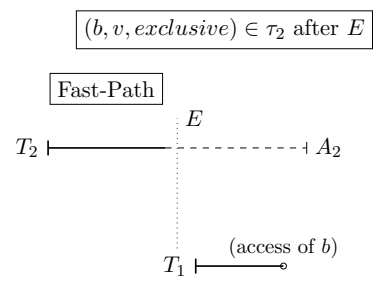

(a) $\tau_{2}$ is invalidated by (fast-path or slow-path) transaction $T_{1}$ 's access of base object $b$

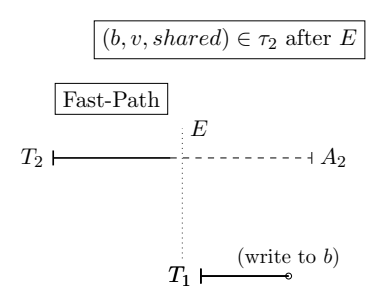

(b) $\tau_{2}$ is invalidated by (fast-path or slow-path) transaction $T_{1}$ 's write to base object $b$

Figure 1: Tracking set aborts in fast-path transactions; we denote a fast-path (and resp. slowpath) transaction by $F$ (and resp. $S$ )

Note that HTM may also abort spuriously, or because of unsupported operations [31]. The first cause can be modelled probabilistically in the above framework, which would not however significantly affect our claims and proofs, except for a more cumbersome presentation. Also, our lower bounds are based exclusively on executions containing t-reads and t-writes. Therefore, in the following, we only consider contention and capacity aborts.

Slow-path and fast-path transactions. In the following, we partition HyTM transactions into fast-path transactions and slow-path transactions. Practically, two separate algorithms (fast-path one and slow-path one) are provided for each t-operation.

A slow-path transaction models a regular software transaction. An event of a slow-path transaction is either an invocation or response of a t-operation, or a rmw primitive on a base object.

A fast-path transaction essentially encapsulates a hardware transaction. An event of a fastpath transaction is either an invocation or response of a t-operation, a cached primitive on a base object, or a cache-commit: $t$-read and t-write are only allowed to contain cached primitives, and try $C$ consists of invoking cache-commit. Furthermore, we assume that a fast-path transaction $T_{k}$ returns $A_{k}$ as soon an underlying cached primitive or cache-commit returns $\perp$. Figure 1 depicts such a scenario illustrating a tracking set abort: fast-path transaction $T_{2}$ executed by process $p_{2}$ accesses a base object $b$ in shared (and resp. exclusive) mode and it is added to its tracking set $\tau_{2}$. Immediately after the access of $b$ by $T_{2}$, a concurrent transaction $T_{1}$ applies a nontrivial primitive to $b$ (and resp. accesses $b$ ). Thus, the tracking of $p_{2}$ is invalidated and $T_{2}$ must be aborted in any extension of this execution.

We provide two key observations on this model regarding the interactions of non-committed fast path transactions with other transactions. Let $E$ be any execution of a HyTM implementation $\mathcal{M}$ in which a fast-path transaction $T_{k}$ is either t-incomplete or aborted. Then the sequence of events $E^{\prime}$ derived by removing all events of $E \mid k$ from $E$ is an execution $\mathcal{M}$. Moreover:

Observation 1. To every slow-path transaction $T_{m} \in \operatorname{txns}(E), E$ is indistinguishable from $E^{\prime}$.

Observation 2. If a fast-path transaction $T_{m} \in \operatorname{txns}(E) \backslash\left\{T_{k}\right\}$ does not incur a tracking set abort in $E$, then $E$ is indistinguishable to $T_{m}$ from $E^{\prime}$.

Intuitively, these observations say that fast-path transactions which are not yet committed are invisible to slow-path transactions, and can communicate with other fast-path transactions only by incurring their tracking-set aborts. Figure 2 illustrates Observation 1 1 a fast-path transaction $T_{2}$ is concurrent to a slow-path transaction $T_{1}$ in an execution $E$. Since $T_{2}$ is t-incomplete or aborted in this execution, $E$ is indistinguishable to $T_{1}$ from an execution $E^{\prime}$ derived by removing all events of $T_{2}$ from $E$. Analogously, to illustrate Observation 2 , if $T_{1}$ is a 


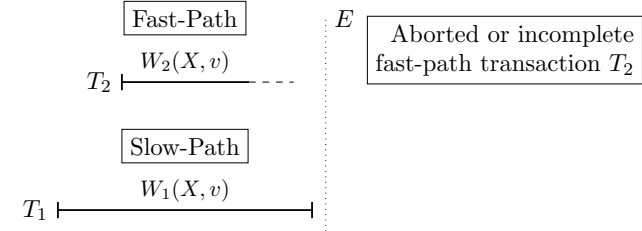

(a)

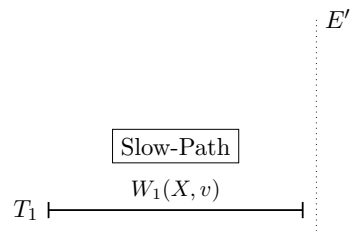

(b)

Figure 2: Execution $E$ in Figure 2a is indistinguishable to $T_{1}$ from the execution $E^{\prime}$ in Figure $2 \mathrm{~b}$

fast-path transaction that does not incur a tracking set abort in $E$, then $E$ is indistinguishable to $T_{1}$ from $E^{\prime}$.

\section{Instrumentation}

Now we define the notion of code instrumentation in fast-path transactions.

An execution $E$ of a HyTM $\mathcal{M}$ appears t-sequential to a transaction $T_{k} \in \operatorname{txns}(E)$ if there exists an execution $E^{\prime}$ of $\mathcal{M}$ such that:

- $\operatorname{txns}\left(E^{\prime}\right) \subseteq \operatorname{txns}(E) \backslash\left\{T_{k}\right\}$ and the configuration after $E^{\prime}$ is t-quiescent,

- every transaction $T_{m} \in \operatorname{txns}(E)$ that precedes $T_{k}$ in real-time order is included in $E^{\prime}$ such that $E\left|m=E^{\prime}\right| m$,

- for every transaction $T_{m} \in \operatorname{txns}\left(E^{\prime}\right), \operatorname{Rset}_{E^{\prime}}\left(T_{m}\right) \subseteq \operatorname{Rset}_{E}\left(T_{m}\right)$ and $\operatorname{Wset}_{E^{\prime}}\left(T_{m}\right) \subseteq \operatorname{Wset}_{E}\left(T_{m}\right)$, and

- $E^{\prime} \cdot E \mid k$ is an execution of $\mathcal{M}$.

Definition 1 (Data and metadata base objects). Let $\mathcal{X}$ be the set of t-objects operated by a HyTM implementation $\mathcal{M}$. Now we partition the set of base objects used by $\mathcal{M}$ into a set $\mathbb{D}$ of data objects and a set $\mathbb{M}$ of metadata objects $(\mathbb{D} \cap \mathbb{M}=\emptyset)$. We further partition $\mathbb{D}$ into sets $\mathbb{D}_{X}$ associated with each t-object $X \in \mathcal{X}: \mathbb{D}=\bigcup_{X \in \mathcal{X}} \mathbb{D}_{X}$, for all $X \neq Y$ in $\mathcal{X}, \mathbb{D}_{X} \cap \mathbb{D}_{Y}=\emptyset$, such that:

1. In every execution $E$, each fast-path transaction $T_{k} \in \operatorname{txns}(E)$ only accesses base objects in $\bigcup_{X \in D \operatorname{Set}\left(T_{k}\right)} \mathbb{D}_{X}$ or $\mathbb{M}$.

2. Let $E \cdot \rho$ and $E \cdot E^{\prime} \cdot \rho^{\prime}$ be two t-complete executions, such that $E$ and $E \cdot E^{\prime}$ are $t$ complete, $\rho$ and $\rho^{\prime}$ are complete executions of a transaction $T_{k} \notin \operatorname{txns}\left(E \cdot E^{\prime}\right), H_{\rho}=H_{\rho^{\prime}}$, and $\forall T_{m} \in \operatorname{txns}\left(E^{\prime}\right), \operatorname{Dset}\left(T_{m}\right) \cap \operatorname{Dset}\left(T_{k}\right)=\emptyset$. Then the states of the base objects $\cup \mathbb{D}_{X}$ in the configuration after $E \cdot \rho$ and $E \cdot E^{\prime} \cdot \rho^{\prime}$ are the same. $X \in D$ Set $\left(T_{k}\right)$

3. Let execution $E$ appear t-sequential to a transaction $T_{k}$ and let the enabled event e of $T_{k}$ after $E$ be a primitive on a base object $b \in \mathbb{D}$. Then, unless e returns $\perp, E \cdot e$ also appears t-sequential to $T_{k}$.

Intuitively, the first condition says that a transaction is only allowed to access data objects based on its data set. The second condition says that transactions with disjoint data sets can communicate only via metadata objects. Finally, the last condition means that base objects in $\mathbb{D}$ may only contain the "values" of t-objects, and cannot be used to detect concurrent transactions. Note that our results will lower bound the number of metadata objects that must be accessed under particular assumptions, thus from a cost perspective, $\mathbb{D}$ should be made as large as possible.

All HyTM proposals we aware of, such as HybridNOrec [9, 32, PhTM [28] and others [11,26], conform to our definition of instrumentation in fast-path transactions. For instance, HybridNOrec [9,32] employs a distinct base object in $\mathbb{D}$ for each t-object and a global sequence lock as 


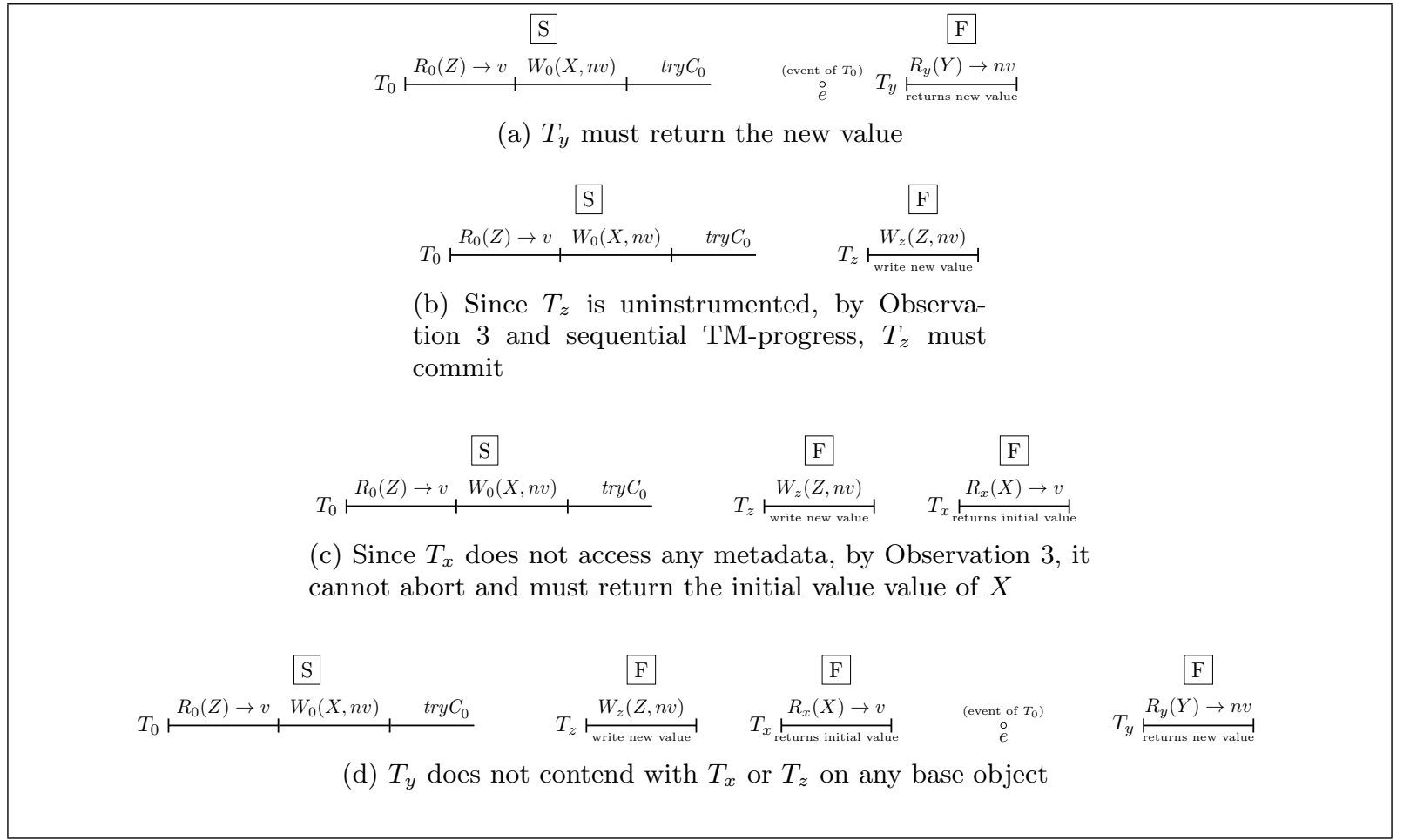

Figure 3: Executions in the proof of Theorem 4 , execution in $3 \mathrm{~d}$ is not strictly serializable

the metadata that is accessed by fast-path transactions to detect concurrency with slow-path transactions. Similarly, the HyTM implementation by Damron et al. [11] also associates a distinct base object in $\mathbb{D}$ for each t-object and additionally, a transaction header and ownership record as metadata base objects.

Definition 2 (Uninstrumented HyTMs). A HyTM implementation $\mathcal{M}$ provides uninstrumented writes (resp. reads) if in every execution $E$ of $\mathcal{M}$, for every write-only (resp. read-only) fastpath transaction $T_{k}$, all primitives in $E \mid k$ are performed on base objects in $\mathbb{D}$. A HyTM is uninstrumented if both its reads and writes are uninstrumented.

Observation 3. Consider any execution $E$ of a HyTM implementation $\mathcal{M}$ which provides uninstrumented reads (resp. writes). For any fast-path read-only (resp. write-only) transaction $T_{k} \notin \operatorname{txns}(E)$, that runs step-contention free after $E$, the execution $E$ appears $t$-sequential to $T_{k}$.

\section{Impossibility of uninstrumented HyTMs}

In this section, we show that any strictly serializable HyTM must be instrumented, even under a very weak progress assumption by which a transaction is guaranteed to commit only when run t-sequentially:

Definition 3 (Sequential TM-progress). A HyTM implementation $\mathcal{M}$ provides sequential TMprogress for fast-path transactions (and resp. slow-path) if in every execution $E$ of $\mathcal{M}$, a fastpath (and resp. slow-path) transaction $T_{k}$ returns $A_{k}$ in $E$ only if $T_{k}$ incurs a capacity abort or $T_{k}$ is concurrent to another transaction. We say that $\mathcal{M}$ provides sequential TM-progress if it provides sequential TM-progress for fast-path and slow-path transactions.

Theorem 4. There does not exist a strictly serializable uninstrumented HyTM implementation that ensures sequential TM-progress and TM-liveness. 
Proof. Suppose by contradiction that such a HyTM $\mathcal{M}$ exists. For simplicity, assume that $v$ is the initial value of t-objects $X, Y$ and $Z$. Let $E$ be the t-complete step contention-free execution of a slow-path transaction $T_{0}$ that performs $\operatorname{read}_{0}(Z) \rightarrow v$, write $_{0}(X, n v)$, write $e_{0}(Y, n v)(n v \neq v)$, and commits. Such an execution exists since $\mathcal{M}$ ensures sequential TM-progress.

By Observation 3, any transaction that runs step contention-free starting from a prefix of $E$ must return a non-abort value. Since any such transaction reading $X$ or $Y$ must return $v$ when it starts from the empty prefix of $E$ and $n v$ when it starts from $E$.

Thus, there exists $E^{\prime}$, the longest prefix of $E$ that cannot be extended with the t-complete step contention-free execution of a fast-path transaction reading $X$ or $Y$ and returning $n v$. Let $e$ is the enabled event of $T_{0}$ in the configuration after $E^{\prime}$. Without loss of generality, suppose that there exists an execution $E^{\prime} \cdot e \cdot E_{y}$ where $E_{y}$ is the t-complete step contention-free execution fragment of some fast-path transaction $T_{y}$ that reads $Y$ is returns $n v$ (Figure 3a).

Claim 5. $\mathcal{M}$ has an execution $E^{\prime} \cdot E_{z} \cdot E_{x}$, where

- $E_{z}$ is the t-complete step contention-free execution fragment of a fast-path transaction $T_{z}$ that writes $n v \neq v$ to $Z$ and commits

- $E_{x}$ is the t-complete step contention-free execution fragment of a fast-path transaction $T_{x}$ that performs a single t-read read $(X) \rightarrow v$ and commits.

Proof. By Observation 3, the extension of $E^{\prime}$ in which $T_{z}$ writes to $Z$ and tries to commit appears t-sequential to $T_{z}$. By sequential TM-progress, $T_{z}$ complets the write and commits. Let $E^{\prime} \cdot E_{z}$ (Figure $3 \mathrm{~b}$ ) be the resulting execution of $\mathcal{M}$.

Similarly, the extension of $E^{\prime}$ in which $T_{x}$ reads $X$ and tries to commit appears t-sequential to $T_{x}$. By sequential TM-progress, $T_{x}$ commits and let $E^{\prime} \cdot E_{x}$ be the resulting execution of $\mathcal{M}$. By the definition of $E^{\prime}, \operatorname{read}_{x}(X)$ must return $v$ in $E^{\prime} \cdot E_{x}$.

Since $\mathcal{M}$ is uninstrumented and the data sets of $T_{x}$ and $T_{z}$ are disjoint, the sets of base objects accessed in the execution fragments $E_{x}$ and $E_{y}$ are also disjoint. Thus, $E^{\prime} \cdot E_{z} \cdot E_{x}$ is indistinguishable to $T_{x}$ from the execution $E^{\prime} \cdot E_{x}$, which implies that $E^{\prime} \cdot E_{z} \cdot E_{x}$ is an execution of $\mathcal{M}$ (Figure $3 \mathrm{c}$ ).

Finally, we prove that the sequence of events, $E^{\prime} \cdot E_{z} \cdot E_{x} \cdot e \cdot E_{y}$ is an execution of $\mathcal{M}$.

Since the transactions $T_{x}, T_{y}, T_{z}$ have pairwise disjoint data sets in $E^{\prime} \cdot E_{z} \cdot E_{x} \cdot e \cdot E_{y}$, no base object accessed ib $E_{y}$ can be accessed in $E_{x}$ and $E_{z}$. The read operation on $X$ performed by $T_{y}$ in $E^{\prime} \cdot e \cdot E_{y}$ returns $n v$ and, by the definition of $E^{\prime}$ and $e, T_{y}$ must have accessed the base object $b$ modified in the event $e$ by $T_{0}$. Thus, $b$ is not accessed in $E_{x}$ and $E_{z}$ and $E^{\prime} \cdot E_{z} \cdot E_{x} \cdot e$ is an execution of $\mathcal{M}$. Summing up, $E^{\prime} \cdot E_{z} \cdot E_{x} \cdot e \cdot E_{y}$ is indistinguishable to $T_{y}$ from $E^{\prime} \cdot e \cdot E_{y}$, which implies that $E^{\prime} \cdot E_{z} \cdot E_{x} \cdot e \cdot E_{y}$ is an execution of $\mathcal{M}$ (Figure 3d).

But the resulting execution is not strictly serializable. Indeed, suppose that a serialization exists. As the value written by $T_{0}$ is returned by a committed transaction $T_{y}, T_{0}$ must be committed and precede $T_{y}$ in the serialization. Since $T_{x}$ returns the initial value of $X, T_{x}$ must precede $T_{0}$. Since $T_{0}$ reads the initial value of $Z, T_{0}$ must precede $T_{z}$. Finally, $T_{z}$ must precede $T_{x}$ to respect the real-time order. The cycle in the serialization establishes a contradiction.

\section{Providing concurrency in HyTM}

In this section, we show that giving HyTM the ability to run and commit transactions in parallel brings considerable instrumentation costs. We focus on a natural progress condition called progressiveness [17-19] that allows a transaction to abort only if it experiences a readwrite or write-write conflict with a concurrent transaction:

Definition 4 (Progressiveness). Transactions $T_{i}$ and $T_{j}$ conflict in an execution $E$ on a $t$-object $X$ if $X \in \operatorname{Dset}\left(T_{i}\right) \cap \operatorname{Dset}\left(T_{j}\right)$ and $X \in W \operatorname{Wet}\left(T_{i}\right) \cup W \operatorname{Wet}\left(T_{j}\right)$. A HyTM implementation $\mathcal{M}$ is fast-path (resp. slow-path) progressive if in every execution $E$ of $\mathcal{M}$ and for every fast-path (and resp. slow-path) transaction $T_{i}$ that aborts in $E$, either $A_{i}$ is a capacity abort or $T_{i}$ conflicts 
with some transaction $T_{j}$ that is concurrent to $T_{i}$ in $E$. We say $\mathcal{M}$ is progressive if it is both fast-path and slow-path progressive.

\subsection{A linear lower bound on instrumentation}

We show that for every opaque fast-path progressive HyTM that provides obstruction-free TMliveness, an arbitrarily long read-only transaction might access a number of distinct metadata base objects that is linear in the size of its read set or experience a capacity abort.

The following auxiliary results will be crucial in proving our lower bound. We observe first that a fast path transaction in a progressive HyTM can contend on a base object only with a non-conflicting transaction.

Lemma 6. Let $\mathcal{M}$ be any fast-path progressive HyTM implementation. Let $E \cdot E_{1} \cdot E_{2}$ be an execution of $\mathcal{M}$ where $E_{1}$ (and resp. $E_{2}$ ) is the step contention-free execution fragment of transaction $T_{1} \notin$ txns $(E)$ (and resp. $T_{2} \notin$ txns $(E)$ ), $T_{1}$ (and resp. $T_{2}$ ) does not conflict with any transaction in $E \cdot E_{1} \cdot E_{2}$, and at least one of $T_{1}$ or $T_{2}$ is a fast-path transaction. Then, $T_{1}$ and $T_{2}$ do not contend on any base object in $E \cdot E_{1} \cdot E_{2}$.

Proof. Suppose, by contradiction that $T_{1}$ or $T_{2}$ contend on the same base object in $E \cdot E_{1} \cdot E_{2}$.

If in $E_{1}, T_{1}$ performs a nontrivial event on a base object on which they contend, let $e_{1}$ be the last event in $E_{1}$ in which $T_{1}$ performs such an event to some base object $b$ and $e_{2}$, the first event in $E_{2}$ that accesses $b$. Otherwise, $T_{1}$ only performs trivial events in $E_{1}$ to base objects on which it contends with $T_{2}$ in $E \cdot E_{1} \cdot E_{2}$ : let $e_{2}$ be the first event in $E_{2}$ in which $E_{2}$ performs a nontrivial event to some base object $b$ on which they contend and $e_{1}$, the last event of $E_{1}$ in $T_{1}$ that accesses $b$.

Let $E_{1}^{\prime}$ (and resp. $E_{2}^{\prime}$ ) be the longest prefix of $E_{1}$ (and resp. $E_{2}$ ) that does not include $e_{1}$ (and resp. $e_{2}$ ). Since before accessing $b$, the execution is step contention-free for $T_{1}, E \cdot E_{1}^{\prime} \cdot E_{2}^{\prime}$ is an execution of $\mathcal{M}$. By construction, $T_{1}$ and $T_{2}$ do not conflict in $E \cdot E_{1}^{\prime} \cdot E_{2}^{\prime}$. Moreover, $E \cdot E_{1} \cdot E_{2}^{\prime}$ is indistinguishable to $T_{2}$ from $E \cdot E_{1}^{\prime} \cdot E_{2}^{\prime}$. Hence, $T_{1}$ and $T_{2}$ are poised to apply contending events $e_{1}$ and $e_{2}$ on $b$ in the execution $\tilde{E}=E \cdot E_{1}^{\prime} \cdot E_{2}^{\prime}$. Recall that at least one event of $e_{1}$ and $e_{2}$ must be nontrivial.

Consider the execution $\tilde{E} \cdot e_{1} \cdot e_{2}^{\prime}$ where $e_{2}^{\prime}$ is the event of $p_{2}$ in which it applies the primitive of $e_{2}$ to the configuration after $\tilde{E} \cdot e_{1}$. After $\tilde{E} \cdot e_{1}, b$ is contained in the tracking set of process $p_{1}$. If $b$ is contained in $\tau_{1}$ in the shared mode, then $e_{2}^{\prime}$ is a nontrivial primitive on $b$, which invalidates $\tau_{1}$ in $\tilde{E} \cdot e_{1} \cdot e_{2}^{\prime}$. If $b$ is contained in $\tau_{1}$ in the exclusive mode, then any subsequent access of $b$ invalidates $\tau_{1}$ in $\tilde{E} \cdot e_{1} \cdot e_{2}^{\prime}$. In both cases, $\tau_{1}$ is invalidated and $T_{1}$ incurs a tracking set abort. Thus, transaction $T_{1}$ must return $A_{1}$ in any extension of $E \cdot e_{1} \cdot e_{2}$-a contradiction to the assumption that $\mathcal{M}$ is progressive.

Iterative application of Lemma 6 implies the following:

Corollary 7. Let $\mathcal{M}$ be any fast-path progressive HyTM implementation. Let $E \cdot E_{1} \cdots E_{i}$. $E_{i+1} \cdots E_{m}$ be any execution of $\mathcal{M}$ where $E_{i}$ is the step contention-free execution fragment of transaction $T_{i} \notin$ txns $(E)$, for all $i \in\{1, \ldots, m\}$ and any two transactions in $E_{1} \cdots E_{m}$ do not conflict. For all $i, j=1, \ldots, m, i \neq j$, if $T_{i}$ is fast-path, then $T_{i}$ and $T_{j}$ do not contend on a base object in $E \cdot E_{1} \cdots E_{m} \cdots E_{m}$

Proof. Let $T_{i}$ be a fast-path transaction. By Lemma 6 , in $E \cdot E_{1} \cdots E_{i} \cdots E_{m}, T_{i}$ does not contend with $T_{i-1}$ (if $i>1$ ) or $T_{i+1}$ (if $i<m$ ) on any base object and, thus, $E_{i}$ commutes with $E_{i-1}$ and $E_{i+1}$. Thus, $E \cdot E_{1} \cdots E_{i-2} \cdot E_{i} \cdot E_{i-1} \cdot E_{i+1} \cdots E_{m}$ (if $i>1$ ) and $E \cdot E_{1} \cdots E_{i-1} \cdot E_{i+1} \cdot E_{i} \cdot E_{i+2} \cdots E_{m}$ (if $i<m$ ) are executions of $\mathcal{M}$. By iteratively applying Lemma 6, we derive that $T_{i}$ does not contend with any $T_{j}, j \neq i$.

Recall that execution fragments $E$ and $E^{\prime}$ are called similar if they export equivalent histories, i.e., no process can see the difference between them by looking at the invocations and 
responses of t-operations. We now use Corollary 7 to show that t-operations only accessing data base objects cannot detect contention with non-conflicting transactions.

Lemma 8. Let $E$ be any -complete execution of a progressive HyTM implementation $\mathcal{M}$ that provides OF TM-liveness. For any $m \in \mathbb{N}$, consider a set of $m$ executions of $\mathcal{M}$ of the form $E \cdot E_{i} \cdot \gamma_{i} \cdot \rho_{i}$ where $E_{i}$ is the $t$-complete step contention-free execution fragment of a transaction $T_{m+i}, \gamma_{i}$ is a complete step contention-free execution fragment of a fast-path transaction $T_{i}$ such that $\operatorname{Dset}\left(T_{i}\right) \cap \operatorname{Dset}\left(T_{m+i}\right)=\emptyset$ in $E \cdot E_{i} \cdot \gamma_{i}$, and $\rho_{i}$ is the execution fragment of a $t$-operation by $T_{i}$ that does not contain accesses to any metadata base object. If, for all $i, j \in\{1, \ldots, m\}, i \neq j$, $\operatorname{Dset}\left(T_{i}\right) \cap \operatorname{Dset}\left(T_{m+j}\right)=\emptyset, \operatorname{Dset}\left(T_{i}\right) \cap \operatorname{Dset}\left(T_{j}\right)=\emptyset$ and $\operatorname{Dset}\left(T_{m+i}\right) \cap \operatorname{Dset}\left(T_{m+j}\right)=\emptyset$, then there exists a t-complete step contention-free execution fragment $E^{\prime}$ that is similar to $E_{1} \cdots E_{m}$ such that for all $i \in\{1, \ldots, m\}, E \cdot E^{\prime} \cdot \gamma_{i} \cdot \rho_{i}$ is an execution of $\mathcal{M}$.

Proof. Observe that any two transactions in the execution fragment $E_{1} \cdots E_{m}$ access mutually disjoint data sets. Since $\mathcal{M}$ is progressive and provides OF TM-liveness, there exists a tsequential execution fragment $E^{\prime}=E_{1}^{\prime} \cdots E_{m}^{\prime}$ such that, for all $i \in\{1, \ldots, m\}$, the execution fragments $E_{i}$ and $E_{i}^{\prime}$ are similar and $E \cdot E^{\prime}$ is an execution of $\mathcal{M}$. Corollary 7 implies that, for all for all $i \in\{1, \ldots, m\}, \mathcal{M}$ has an execution of the form $E \cdot E_{1}^{\prime} \cdots E_{i}^{\prime} \cdots E_{m}^{\prime} \cdot \gamma_{i}$. More specifically, $\mathcal{M}$ has an execution of the form $E \cdot \gamma_{i} \cdot E_{1}^{\prime} \cdots E_{i}^{\prime} \cdots E_{m}^{\prime}$. Recall that the execution fragment $\rho_{i}$ of fast-path transaction $T_{i}$ that extends $\gamma_{i}$ contains accesses only to base objects in $\bigcup_{X \in D S t\left(T_{i}\right)} \mathbb{D}_{X}$. Moreover, for all $i, j \in\{1, \ldots, m\} ; i \neq j, \operatorname{Dset}\left(T_{i}\right) \cap \operatorname{Dset}\left(T_{m+j}\right)=\emptyset$ and $X \in D \operatorname{Set}\left(T_{i}\right)$

$\operatorname{Dset}\left(T_{m+i}\right) \cap \operatorname{Dset}\left(T_{m+j}\right)=\emptyset$.

It follows that $\mathcal{M}$ has an execution of the form $E \cdot \gamma_{i} \cdot E_{1}^{\prime} \cdots E_{i}^{\prime} \cdot \rho_{i} \cdot E_{i+1}^{\prime} \cdots E_{m}^{\prime}$. and the states of each of the base objects $\bigcup_{X \in D \operatorname{Set}\left(T_{i}\right)} \mathbb{D}_{X}$ accessed by $T_{i}$ in the configuration after $E \cdot \gamma_{i} \cdot E_{1}^{\prime} \cdots E_{i}^{\prime}$ and $E \cdot \gamma_{i} \cdot E_{i}$ are the same. But $E \cdot \gamma_{i} \cdot E_{i} \cdot \rho_{i}$ is an execution of $\mathcal{M}$. Thus, for all $i \in\{1, \ldots, m\}$, $\mathcal{M}$ has an execution of the form $E \cdot E^{\prime} \cdot \gamma_{i} \cdot \rho_{i}$.

Finally, we are now ready to derive our lower bound.

Let $\kappa$ be the smallest integer such that some fast-path transaction running step contentionfree after a t-complete execution performs $\kappa$ t-reads and incurs a capacity abort. In other words, if a fast-path transaction reads less than $\kappa$ t-objects, it cannot incur a capacity abort.

We prove that, for all $m \leq \kappa-1$, there exists a t-complete execution $E_{m}$ and a set $S_{m}$ $\left(\left|S_{m}\right|=2^{\kappa-m}\right)$ of read-only fast-path transactions such that (1) each transaction in $S_{m}$ reads $m$ t-objects, (2) the data sets of any two transactions in $S_{m}$ are disjoint, (3) in the step contentionfree execution of any transaction in $S_{m}$ extending $E_{m}$, every t-read accesses at least one distinct metadata base object.

By induction, we assume that the induction statement holds for all $m<\kappa-1$ (the base case $m=0$ is trivial) and prove that $E_{m+1}$ and $S_{m+1}$ satisfying the condition above exist. Pick any two transactions from the set $S_{m}$. We construct $E_{m}^{\prime}$, a t-complete extension of $E_{m}$ by the execution of a slow-path transaction writing to two distinct t-objects $X$ and $Y$, such that the two picked transactions, running step contention-free after that, cannot distinguish $E_{m}$ and $E_{m}^{\prime}$. Now we let each of the transactions read one of the two t-objects $X$ and $Y$. We show that at least one of them must access a new metadata base object in this $(m+1)^{t h}$ t-read (otherwise, the resulting execution would not be opaque). By repeating this argument for each pair of transactions, we derive that there exists $E_{m+1}$, a t-complete extension of $E_{m}$, such that at least half of the transaction in $S_{m}$ must access a new distinct metadata base object in its $(m+1)^{t h}$ t-read when it runs t-sequentially after $E_{m+1}$. Intuitively, we construct $E_{m+1}$ by "gluing" all these executions $E_{m}^{\prime}$ together, which is possible thanks to Lemma 6 . These transactions constitute $S_{m+1} \subset S_{m},\left|S_{m+1}\right|=\left|S_{m}\right| / 2=2^{\kappa-(m+1)}$.

Theorem 9. Let $\mathcal{M}$ be any progressive, opaque HyTM implementation that provides OF TMliveness. For every $m \in \mathbb{N}$, there exists an execution $E$ in which some fast-path read-only 
transaction $T_{k} \in$ txns $(E)$ satisfies either (1) Dset $\left(T_{k}\right) \leq m$ and $T_{k}$ incurs a capacity abort in $E$ or (2) Dset $\left(T_{k}\right)=m$ and $T_{k}$ accesses $\Omega(m)$ distinct metadata base objects in $E$.

Here is a high-level overview of the proof technique. Let $\kappa$ be the smallest integer such that some fast-path transaction running step contention-free after a t-quiescent configuration performs $\kappa$ t-reads and incurs a capacity abort.

We prove that, for all $m \leq \kappa-1$, there exists a t-complete execution $E_{m}$ and a set $S_{m}$ with $\left|S_{m}\right|=2^{\kappa-m}$ of read-only fast-path transactions that access mutually disjoint data sets such that each transaction in $S_{m}$ that runs step contention-free from $E_{m}$ and performs t-reads of $m$ distinct t-objects accesses at least one distinct metadata base object within the execution of each t-read operation.

We proceed by induction. Assume that the induction statement holds for all $m<k a p p a-1$. We prove that a set $S_{m+1} ;\left|S_{m+1}\right|=2^{\kappa-(m+1)}$ of fast-path transactions, each of which run step contention-free after the same t-complete execution $E_{m+1}$, perform $m+1$ t-reads of distinct t-objects so that at least one distinct metadata base object is accessed within the execution of each t-read operation. In our construction, we pick any two new transactions from the set $S_{m}$ and show that one of them running step contention-free from a t-complete execution that extends $E_{m}$ performs $m+1$ t-reads of distinct t-objects so that at least one distinct metadata base object is accessed within the execution of each t-read operation. In this way, the set of transactions is reduced by half in each step of the induction until one transaction remains which must have accessed a distinct metadata base object in every one of its $m+1$ t-reads.

Intuitively, since all the transactions that we use in our construction access mutually disjoint data sets, we can apply Lemma 6 to construct a t-complete execution $E_{m+1}$ such that each of the fast-path transactions in $S_{m+1}$ when running step contention-free after $E_{m+1}$ perform $m+1$ t-reads so that at least one distinct metadata base object is accessed within the execution of each t-read operation.

We now present the formal proof:

Proof. In the constructions which follow, every fast-path transaction executes at most $m+$ 1 t-reads. Let $\kappa$ be the smallest integer such that some fast-path transaction running step contention-free after a t-quiescent configuration performs $\kappa$ t-reads and incurs a capacity abort. We proceed by induction.

Induction statement. We prove that, for all $m \leq \kappa-1$, there exists a t-complete execution $E_{m}$ and a set $S_{m}$ with $\left|S_{m}\right|=2^{\kappa-m}$ of read-only fast-path transactions that access mutually disjoint data sets such that each transaction $T_{f_{i}} \in S_{m}$ that runs step contention-free from $E_{m}$ and performs t-reads of $m$ distinct t-objects accesses at least one distinct metadata base object within the execution of each t-read operation. Let $E_{f_{i}}$ be the step contention-free execution of $T_{f_{i}}$ after $E_{m}$ and let $\operatorname{Dset}\left(T_{f_{i}}\right)=\left\{X_{i, 1}, \ldots, X_{i, m}\right\}$.

The induction. Assume that the induction statement holds for all $m \leq \kappa-1$. The statement is trivially true for the base case $m=0$ for every $\kappa \in \mathbb{N}$.

We will prove that a set $S_{m+1} ;\left|S_{m+1}\right|=2^{\kappa-(m+1)}$ of fast-path transactions, each of which run step contention-free from the same t-quiescent configuration $E_{m+1}$, perform $m+1$ t-reads of distinct t-objects so that at least one distinct metadata base object is accessed within the execution of each t-read operation.

The construction proceeds in phases: there are exactly $\frac{\left|S_{m}\right|}{2}$ phases. In each phase, we pick any two new transactions from the set $S_{m}$ and show that one of them running step contentionfree after a t-complete execution that extends $E_{m}$ performs $m+1$ t-reads of distinct t-objects so that at least one distinct metadata base object is accessed within the execution of each t-read operation.

Throughout this proof, we will assume that any two transactions (and resp. execution fragments) with distinct subscripts represent distinct identifiers. 
For all $i \in\left\{0, \ldots, \frac{\left|S_{m}\right|}{2}-1\right\}$, let $X_{2 i+1}, X_{2 i+2} \notin \bigcup_{i=0}^{\left|S_{m}\right|-1}\left\{X_{i, 1}, \ldots, X_{i, m}\right\}$ be distinct t-objects and let $v$ be the value of $X_{2 i+1}$ and $X_{2 i+2}$ after $E_{m}$. Let $T_{s_{i}}$ denote a slow-path transaction which writes $n v \neq v$ to $X_{2 i+1}$ and $X_{2 i+2}$. Let $E_{s_{i}}$ be the t-complete step contention-free execution fragment of $T_{s_{i}}$ running immediately after $E_{m}$.

Let $E_{s_{i}}^{\prime}$ be the longest prefix of the execution $E_{s_{i}}$ such that $E_{m} \cdot E_{s_{i}}^{\prime}$ can be extended neither with the complete step contention-free execution fragment of transaction $T_{f_{2 i+1}}$ that performs its $m$ t-reads of $X_{2 i+1,1}, \ldots, X_{2 i+1, m}$ and then performs read $_{f_{2 i+1}}\left(X_{2 i+1}\right)$ and returns $n v$, nor with the complete step contention-free execution fragment of some transaction $T_{f_{2 i+2}}$ that performs t-reads of $X_{2 i+2}, \ldots, X_{2 i+2, m}$ and then performs read $f_{f_{2 i+2}}\left(X_{2 i+2}\right)$ and returns $n v$. Progressiveness and OF TM-liveness of $\mathcal{M}$ stipulates that such an execution exists.

Let $e_{i}$ be the enabled event of $T_{s_{i}}$ in the configuration after $E_{m} \cdot E_{s_{i}}^{\prime}$. By construction, the execution $E_{m} \cdot E_{s_{i}}^{\prime}$ can be extended with at least one of the complete step contention-free executions of transaction $T_{f_{2 i+1}}$ performing $(m+1)$ t-reads of $X_{2 i+1,1}, \ldots, X_{2 i+1, m}, X_{2 i+1}$ such that $\operatorname{read}_{f_{2 i+1}}\left(X_{2 i+1}\right) \rightarrow n v$ or transaction $T_{f_{2 i+2}}$ performing t-reads of $X_{2 i+2,1}, \ldots, X_{2 i+2, m}, X_{2 i+2}$ such that read $_{f_{2 i+2}}\left(X_{2 i+2}\right) \rightarrow n v$. Without loss of generality, suppose that $T_{f_{2 i+1}}$ reads the value of $X_{2 i+1}$ to be $n v$ after $E_{m} \cdot E_{0_{i}}^{\prime} \cdot e_{i}$.

For any $i \in\left\{0, \ldots, \frac{\left|S_{m}\right|}{2}-1\right\}$, we will denote by $\alpha_{i}$ the execution fragment which we will construct in phase $i$. For any $i \in\left\{0, \ldots, \frac{\left|S_{m}\right|}{2}-1\right\}$, we prove that $\mathcal{M}$ has an execution of the form $E_{m} \cdot \alpha_{i}$ in which $T_{f_{2 i+1}}$ (or $T_{f_{2 i+2}}$ ) running step contention-free after a t-complete execution that extends $E_{m}$ performs $m+1$ t-reads of distinct t-objects so that at least one distinct metadata base object is accessed within the execution of each first $m$ t-read operations and $T_{f_{2 i+1}}$ (or $T_{f_{2 i+2}}$ ) is poised to apply an event after $E_{m} \cdot \alpha_{i}$ that accesses a distinct metadata base object during the $(m+1)^{t h}$ t-read. Furthermore, we will show that $E_{m} \cdot \alpha_{i}$ appears t-sequential to $T_{f_{2 i+1}}\left(\right.$ or $\left.T_{f_{2 i+2}}\right)$.

(Construction of phase i)

Let $E_{f_{2 i+1}}$ (and resp. $E_{f_{2 i+2}}$ ) be the complete step contention-free execution of the t-reads of $X_{2 i+1,1}, \ldots, X_{2 i+1, m}$ (and resp. $X_{2 i+2,1}, \ldots, X_{2 i+2, m}$ ) running after $E_{m}$ by $T_{f_{2 i+1}}$ (and resp. $T_{f_{2 i+2}}$ ). By the inductive hypothesis, transaction $T_{f_{2 i+1}}$ (and resp. $T_{f_{2 i+2}}$ ) accesses $m$ distinct metadata objects in the execution $E_{m} \cdot E_{f_{2 i+1}}$ (and resp. $E_{m} \cdot E_{f_{2 i+2}}$ ). Recall that transaction $T_{f_{2 i+1}}$ does not conflict with transaction $T_{s_{i}}$. Thus, by Corollary $7, \mathcal{M}$ has an execution of the form $E_{m} \cdot E_{s_{i}}^{\prime} \cdot e_{i} \cdot E_{f_{2 i+1}}$ (and resp. $E_{m} \cdot E_{s_{i}}^{\prime} \cdot e_{i} \cdot E_{f_{2 i+2}}$ ).

Let $E_{r f_{2 i+1}}$ be the complete step contention-free execution fragment of $\operatorname{read}_{f_{2 i+1}}\left(X_{2 i+1}\right)$ that extends $E_{2 i+1}=E_{m} \cdot E_{s_{i}}^{\prime} \cdot e_{i} \cdot E_{f_{2 i+1}}$. By OF TM-liveness, read $_{f_{2 i+1}}\left(X_{2 i+1}\right)$ must return a matching response in $E_{2 i+1} \cdot E_{r f_{2 i+1}}$. We now consider two cases.

Case I: Suppose $E_{r f_{2 i+1}}$ accesses at least one metadata base object b not previously accessed by $T_{f_{2 i+1}}$.

Let $E_{r f_{2 i+1}}^{\prime}$ be the longest prefix of $E_{r f_{2 i+1}}$ which does not apply any primitives to any metadata base object $b$ not previously accessed by $T_{f_{2 i+1}}$. The execution $E_{m} \cdot E_{s_{i}}^{\prime} \cdot e_{i} \cdot E_{f_{2 i+1}} \cdot E_{r f_{2 i+1}}^{\prime}$ appears t-sequential to $T_{f_{2 i+1}}$ because $E_{f_{2 i+1}}$ does not contend with $T_{s_{i}}$ on any base object and any common base object accessed in the execution fragments $E_{r x_{2 i+1}}^{\prime}$ and $E_{s_{i}}$ by $T_{f_{2 i+1}}$ and $T_{s_{i}}$ respectively must be data objects contained in $\mathbb{D}$. Thus, we have that $\left|\operatorname{Dset}\left(T_{f_{2 i+1}}\right)\right|=m+1$ and that $T_{f_{2 i+1}}$ accesses $m$ distinct metadata base objects within each of its first $m$ t-read operations and is poised to access a distinct metadata base object during the execution of the $(m+1)^{t h}$ t-read. In this case, let $\alpha_{i}=E_{m} \cdot E_{s_{i}}^{\prime} \cdot e_{i} \cdot E_{f_{2 i+1}} \cdot E_{r f_{2 i+1}}^{\prime}$.

Case II: Suppose $E_{r f_{2 i+1}}$ does not access any metadata base object not previously accessed by $T_{f_{2 i+1}}$.

In this case, we will first prove the following:

Claim 10. $\mathcal{M}$ has an execution of the form $E_{2 i+2}=E_{m} \cdot E_{s_{i}}^{\prime} \cdot e_{i} \cdot \bar{E}_{f_{2 i+1}} \cdot E_{f_{2 i+2}}$ where $\bar{E}_{f_{2 i+1}}$ is the $t$-complete step contention-free execution of $T_{f_{2 i+1}}$ in which read $f_{f_{2 i+1}}\left(X_{2 i+1}\right) \rightarrow n v, T_{f_{2 i+1}}$ invokes try $C_{f_{2 i+1}}$ and returns a matching response. 
Proof. Since $E_{r f_{2 i+1}}$ does not contain accesses to any distinct metadata base objects, the execution $E_{m} \cdot E_{s_{i}}^{\prime} \cdot e_{i} \cdot E_{f_{2 i+1}} \cdot E_{r f_{2 i+1}}$ appears t-sequential to $T_{f_{2 i+1}}$. By definition of the event $e_{i}$, $\operatorname{read}_{f_{2 i+1}}\left(X_{2 i+1}\right)$ must access the base object to which the event $e_{i}$ applies a nontrivial primitive and return the response $n v$ in $E_{s_{i}}^{\prime} \cdot e_{i} \cdot E_{f_{2 i+1}} \cdot E_{r f_{2 i+1}}$. By OF TM-liveness, it follows that $E_{m} \cdot E_{s_{i}}^{\prime} \cdot e_{i} \cdot \bar{E}_{f_{2 i+1}}$ is an execution of $\mathcal{M}$.

Now recall that $E_{m} \cdot E_{s_{i}}^{\prime} \cdot e_{i} \cdot E_{f_{2 i+2}}$ is an execution of $\mathcal{M}$ because transactions $T_{f_{2 i+2}}$ and $T_{s_{i}}$ do not conflict in this execution and thus, cannot contend on any base object. Finally, because $T_{f_{2 i+1}}$ and $T_{f_{2 i+2}}$ access disjoint data sets in $E_{m} \cdot E_{s_{i}}^{\prime} \cdot e_{i} \cdot \bar{E}_{f_{2 i+1}} \cdot E_{f_{2 i+2}}$, by Lemma 6 again, we have that $E_{m} \cdot E_{s_{i}}^{\prime} \cdot e_{i} \cdot \bar{E}_{f_{2 i+1}} \cdot E_{f_{2 i+2}}$ is an execution of $\mathcal{M}$.

Let $E_{r f_{2 i+2}}$ be the complete step contention-free execution fragment of $\operatorname{read}_{f_{2 i+2}}\left(X_{2 i+2}\right)$ after $E_{m} \cdot E_{s_{i}}^{\prime} \cdot e_{i} \cdot E_{f_{2 i+1}} \cdot E_{f_{2 i+2}}$. By the induction hypothesis and Claim 10, transaction $T_{f_{2 i+2}}$ must access $m$ distinct metadata base objects in the execution $E_{m} \cdot E_{s_{i}}^{\prime} \cdot e_{i} \cdot \bar{E}_{f_{2 i+1}} \cdot E_{f_{2 i+2}}$.

If $E_{r f_{2 i+2}}$ accesses some metadata base object, then by the argument given in Case I applied to transaction $T_{f_{2 i+2}}$, we get that $T_{f_{2 i+2}}$ accesses $m$ distinct metadata base objects within each of the first $m$ t-read operations and is poised to access a distinct metadata base object during the execution of the $(m+1)^{t h}$ t-read.

Thus, suppose that $E_{r f_{2 i+2}}$ does not access any metadata base object previously accessed by $T_{f_{2 i+2}}$. We claim that this is impossible and proceed to derive a contradiction. In particular, $E_{r f_{2 i+2}}$ does not contend with $T_{s_{i}}$ on any metadata base object. Consequently, the execution $E_{m} \cdot E_{s_{i}}^{\prime} \cdot e_{i} \cdot \bar{E}_{f_{2 i+1}} \cdot E_{f_{2 i+2}}$ appears t-sequential to $T_{x_{2 i+2}}$ since $E_{r x_{2 i+2}}$ only contends with $T_{s_{i}}$ on base objects in $\mathbb{D}$. It follows that $E_{2 i+2} \cdot E_{r f_{2 i+2}}$ must also appear t-sequential to $T_{f_{2 i+2}}$ and so $E_{r f_{2 i+2}}$ cannot abort. Recall that the base object, say $b$, to which $T_{s_{i}}$ applies a nontrivial primitive in the event $e_{i}$ is accessed by $T_{f_{2 i+1}}$ in $E_{m} \cdot E_{s_{i}}^{\prime} \cdot e_{i} \cdot \bar{E}_{f_{2 i+1}} \cdot E_{f_{2 i+2}}$; thus, $b \in \mathbb{D}_{X_{2 i+1}}$. Since $X_{2 i+1} \notin D \operatorname{Det}\left(T_{f_{2 i+2}}\right), b$ cannot be accessed by $T_{f_{2 i+2}}$. Thus, the execution $E_{m} \cdot E_{s_{i}}^{\prime} \cdot e_{i}$. $\bar{E}_{f_{2 i+1}} \cdot E_{f_{2 i+2}} \cdot E_{r f_{2 i+2}}$ is indistinguishable to $T_{f_{2 i+2}}$ from the execution $\hat{E}_{i} \cdot E_{s_{i}}^{\prime} \cdot E_{f_{2 i+2}} \cdot E_{r f_{2 i+2}}$ in which read $_{f_{2 i+2}}\left(X_{2 i+2}\right)$ must return the response $v$ (by construction of $E_{s_{i}}^{\prime}$ ).

But we observe now that the execution $E_{m} \cdot E_{s_{i}}^{\prime} \cdot e_{i} \cdot \bar{E}_{f_{2 i+1}} \cdot E_{f_{2 i+2}} \cdot E_{r f_{2 i+2}}$ is not opaque. In any serialization corresponding to this execution, $T_{s_{i}}$ must be committed and must precede $T_{f_{2 i+1}}$ because $T_{f_{2 i+1}}$ read $n v$ from $X_{2 i+1}$. Also, transaction $T_{f_{2 i+2}}$ must precede $T_{s_{i}}$ because $T_{f_{2 i+2}}$ read $v$ from $X_{2 i+2}$. However $T_{f_{2 i+1}}$ must precede $T_{f_{2 i+2}}$ to respect real-time ordering of transactions. Clearly, there exists no such serialization - contradiction.

Letting $E_{r f_{2 i+2}}^{\prime}$ be the longest prefix of $E_{r f_{2 i+2}}$ which does not access a base object $b \in \mathbb{M}$ not previously accessed by $T_{f_{2 i+2}}$, we can let $\alpha_{i}=E_{s_{i}}^{\prime} \cdot e_{i} \cdot \bar{E}_{f_{2 i+1}} \cdot E_{f_{2 i+2}} \cdot E_{r f_{2 i+2}}^{\prime}$ in this case.

Combining Cases I and II, the following claim holds.

Claim 11. For each $i \in\left\{0, \ldots, \frac{\left|S_{m}\right|}{2}-1\right\}, \mathcal{M}$ has an execution of the form $E_{m} \cdot \alpha_{i}$ in which

(1) some fast-path transaction $T_{i} \in \operatorname{txns}\left(\alpha_{i}\right)$ performs $t$-reads of $m+1$ distinct $t$-objects so that at least one distinct metadata base object is accessed within the execution of each of the first $m$ t-reads, $T_{i}$ is poised to access a distinct metadata base object after $E_{m} \cdot \alpha_{i}$ during the execution of the $(m+1)^{\text {th }} t$-read and the execution appears t-sequential to $T_{i}$,

(2) the two fast-path transactions in the execution fragment $\alpha_{i}$ do not contend on the same base object.

(Collecting the phases)

We will now describe how we can construct the set $S_{m+1}$ of fast-path transactions from these $\frac{\left|S_{m}\right|}{2}$ phases and force each of them to access $m+1$ distinct metadata base objects when running step contention-free after the same t-complete execution.

For each $i \in\left\{0, \ldots, \frac{\left|S_{m}\right|}{2}-1\right\}$, let $\beta_{i}$ be the subsequence of the execution $\alpha_{i}$ consisting of all the events of the fast-path transaction that is poised to access a $(m+1)^{t h}$ distinct metadata base object. Henceforth, we denote by $T_{i}$ the fast-path transaction that participates in $\beta_{i}$. Then, from Claim 11, it follows that, for each $i \in\left\{0, \ldots, \frac{\left|S_{m}\right|}{2}-1\right\}, \mathcal{M}$ has an execution of the form $E_{m} \cdot E_{s_{i}}^{\prime} \cdot e_{i} \cdot \beta_{i}$ in which the fast-path transaction $T_{i}$ performs t-reads of $m+1$ distinct t-objects so that at least one distinct metadata base object is accessed within the execution of each of 
the first $m$ t-reads, $T_{i}$ is poised to access a distinct metadata base object after $E_{m} \cdot E_{s_{i}}^{\prime} \cdot e_{i} \cdot \beta_{i}$ during the execution of the $(m+1)^{t h}$ t-read and the execution appears t-sequential to $T_{i}$.

The following result is a corollary to the above claim that is obtained by applying the definition of "appears t-sequential". Recall that $E_{s_{i}}^{\prime} \cdot e_{i}$ is the t-incomplete execution of slowpath transaction $T_{s_{i}}$ that accesses t-objects $X_{2 i+1}$ and $X_{2 i+2}$.

Corollary 12. For all $i \in\left\{0, \ldots, \frac{\mid\left(S_{m} \mid\right.}{2}-1\right\}, \mathcal{M}$ has an execution of the form $E_{m} \cdot E_{i} \cdot \beta_{i}$ such that the configuration after $E_{m} \cdot E_{i}$ is t-quiescent, $\operatorname{txns}\left(E_{i}\right) \subseteq\left\{T_{s_{i}}\right\}$ and $\operatorname{Dset}\left(T_{s_{i}}\right) \subseteq\left\{X_{2 i+1}, X_{2 i+2}\right\}$ in $E_{i}$.

We can represent the execution $\beta_{i}=\gamma_{i} \cdot \rho_{i}$ where fast-path transaction $T_{i}$ performs complete t-reads of $m$ distinct t-objects in $\gamma_{i}$ and then performs an incomplete t-read of the $(m+1)^{t h}$ t-object in $\rho_{i}$ in which $T_{i}$ only accesses base objects in $\bigcup\{X\}$. Recall that $T_{i}$ and $T_{s_{i}}$ do $X \in \operatorname{DSet}\left(T_{i}\right)$

not contend on the same base object in the execution $E_{m} \cdot E_{i} \cdot \gamma_{i}$. Thus, for all $i \in\left\{0, \ldots, \frac{\left|S_{m}\right|}{2}-1\right\}$, $\mathcal{M}$ has an execution of the form $E_{m} \cdot \gamma_{i} \cdot E_{i} \cdot \rho_{i}$.

Observe that the fast-path transaction $T_{i} \in \gamma_{i}$ does not access any t-object that is accessed by any slow-path transaction in the execution fragment $E_{0} \cdots E_{\frac{\left|S_{m}\right|}{2}-1}$. By Lemma 8, there exists a t-complete step contention-free execution fragment $E^{\prime}$ that is similar to $E_{0} \cdots E_{\frac{\left|S_{m}\right|}{2}-1}$ such that for all $i \in\left\{0, \ldots, \frac{\left|S_{m}\right|}{2}-1\right\}, \mathcal{M}$ has an execution of the form $E_{m} \cdot E^{\prime} \cdot \gamma_{i} \cdot \rho_{i}$. By our construction, the enabled event of each fast-path transaction $T_{i} \in \beta_{i}$ in this execution is an access to a distinct metadata base object.

Let $S_{m+1}$ denote the set of all fast-path transactions that participate in the execution fragment $\beta_{0} \cdots \beta_{\frac{\mid\left(S_{m} \mid\right.}{2}-1}$ and $E_{m+1}=E_{m} \cdot E^{\prime}$. Thus, $\left|S_{m+1}\right|$ fast-path transactions, each of which run step contention-free from the same t-quiescent configuration, perform $m+1$ t-reads of distinct t-objects so that at least one distinct metadata base object is accessed within the execution of each t-read operation. This completes the proof.

\subsection{A matching upper bound}

We prove that the lower bound in Theorem 9 is tight by describing an instrumentationoptimal" HyTM implementation (Algorithm 1) that is opaque, progressive, provides wait-free TM-liveness, uses invisible reads.

Definition 5 (Invisible reads). We say that a HyTM implementation $\mathcal{M}$ uses fast-path (and resp. slow-path) invisible reads if for every execution $E$ of $\mathcal{M}$ and every fast-path (and resp. slow-path) transaction $T_{k} \in$ txns $(E), E \mid k$ does not contain any nontrivial events.

Base objects. For every t-object $X_{j}$, our implementation maintains a base object $v_{j} \in \mathbb{D}$ that stores the value of $X_{j}$ and a metadata base object $r_{j}$, which is a lock bit that stores 0 or 1 .

Fast-path transactions. For a fast-path transaction $T_{k}$, the $\operatorname{read}_{k}\left(X_{j}\right)$ implementation first reads $r_{j}$ to check if $X_{j}$ is locked by a concurrent updating transaction. If so, it returns $A_{k}$, else it returns the value of $X_{j}$. Updating fast-path transactions use uninstrumented writes: write $\left(X_{j}, v\right)$ simply stores the cached state of $X_{j}$ along with its value $v$ and if the cache has not been invalidated, updates the shared memory during $\operatorname{try} C_{k}$ by invoking the commit-cache primitive.

Slow-path read-only transactions. Any $\operatorname{read}_{k}\left(X_{j}\right)$ invoked by a slow-path transaction first reads the value of the object from $v_{j}$, checks if $r_{j}$ is set and then performs value-based validation on its entire read set to check if any of them have been modified. If either of these conditions is true, the transaction returns $A_{k}$. Otherwise, it returns the value of $X_{j}$. A read-only transaction simply returns $C_{k}$ during the tryCommit. 
Slow-path updating transactions. The write $_{k}(X, v)$ implementation of a slow-path transaction stores $v$ and the current value of $X_{j}$ locally, deferring the actual update in shared memory to tryCommit.

During $\operatorname{try} C_{k}$, an updating slow-path transaction $T_{k}$ attempts to obtain exclusive write access to its entire write set as follows: for every t-object $X_{j} \in W \operatorname{set}\left(T_{k}\right)$, it writes 1 to each base object $r_{j}$ by performing a compare-and-set (cas) primitive that checks if the value of $r_{j}$ is not 1 and, if so, replaces it with 1 . If the cas fails, then $T_{k}$ releases the locks on all objects $X_{\ell}$ it had previously acquired by writing 0 to $r_{\ell}$ and then returns $A_{k}$. Intuitively, if the cas fails, some concurrent transaction is performing a t-write to a t-object in $W \operatorname{set}\left(T_{k}\right)$. If all the locks on the write set were acquired successfully, $T_{k}$ checks if any t-object in $\operatorname{Rset}\left(T_{k}\right)$ is concurrently being updated by another transaction and then performs value-based validation of the read set. If a conflict is detected from the these checks, the transaction is aborted. Finally, $\operatorname{try} C_{k}$ attempts to write the values of the t-objects via cas operations. If any cas on the individual base objects fails, there must be a concurrent fast-path writer, and so $T_{k}$ rolls back the state of the base objects that were updated, releases locks on its write set and returns $A_{k}$. The roll backs are performed with cas operations, skipping any which fail to allow for concurrent fast-path writes to locked locations. Note that if a concurrent read operation of a fast-path transaction $T_{\ell}$ finds an "invalid" value in $v_{j}$ that was written by such transaction $T_{k}$ but has not been rolled back yet, then $T_{\ell}$ either incurs a tracking set abort later because $T_{k}$ has updated $v_{j}$ or finds $r_{j}$ to be 1. In both cases, the read operation of $T_{\ell}$ aborts.

The implementation uses invisible reads (no nontrivial primitives are applied by reading transactions). Every t-operation returns a matching response within a finite number of its steps.

Complexity. Every t-read operation performed by a fast-path transaction accesses a metadata base object once (the lock bit corresponding to the t-object), which is the price to pay for detecting conflicting updating slow-path transactions. Write operations of fast-path transactions are uninstrumented. Thus:

Theorem 13. There exists an opaque HyTM implementation that provides uninstrumented writes, invisible reads, progressiveness and wait-free TM-liveness such that in its every execution $E$, every read-only fast-path transaction $T \in$ txns $(E)$ accesses $O(|R \operatorname{set}(T)|)$ distinct metadata base objects.

\section{Providing partial concurrency at low cost}

We showed that allowing fast-path transactions to run concurrently in HyTM results in an instrumentation cost that is proportional to the read-set size of a fast-path transaction. But can we run at least some transactions concurrently with constant instrumentation cost, while still keeping invisible reads?

Algorithm 2 implements a slow-path progressive opaque HyTM with invisible reads and wait-free TM-liveness. To fast-path transactions, it only provides sequential TM-progress (they are only guaranteed to commit in the absence of concurrency), but in return the algorithm is only using a single metadata base object $f a$ that is read once by a fast-path transaction and accessed twice with a fetch-and-add primitive by an updating slow-path transaction. Thus, the instrumentation cost of the algorithm is constant.

Intuitively, $f a$ allows fast-path transactions to detect the existence of concurrent updating slow-path transactions. Each time an updating slow-path updating transaction tries to commit, it increments $f a$ and once all writes to data base objects are completed (this part of the algorithm is identical to Algorithm 1) or the transaction is aborted, it decrements $f a$. Therefore, $f a \neq 0$ means that at least one slow-path updating transaction is incomplete. A fast-path transaction simply checks if $f a \neq 0$ in the beginning and aborts if so, otherwise, its code is identical to that 
in Algorithm 1. Note that this way, any update of $f a$ automatically causes a tracking set abort of any incomplete fast-path transaction.

Theorem 14. There exists an opaque HyTM implementation that provides uninstrumented writes, invisible reads, progressiveness for slow-path transactions, sequential TM-progress for fast-path transactions and wait-free TM-liveness such that in every its execution E, every fastpath transaction accesses at most one metadata base object.

\section{Related work}

The notions of opacity and progressiveness for STMs, adopted in this paper for HyTMs, were introduced in [17] and [18, respectively.

Uninstrumented HTMs may be viewed as being inherently disjoint-access parallel, a notion formalized in [7,25]. As such, some of the techniques used in Theorems 4 and 9 resemble those used in 6, 7, 16, 20]. The software component of the HyTM algorithms presented in this paper is inspired by progressive STM implementations like [10,13,27] and is subject to the lower bounds for progressive STMs established in [6, 18, 20,27.

Circa 2005, several papers introduced HyTM implementations [4, 11, 26] that integrated HTMs with variants of DSTM 23. These implementations provide nontrivial concurrency between hardware and software transactions, by instrumenting a hardware transaction's toperations with accesses to metadata to detect conflicting software transactions. Thus, they impose per-access instrumentation overhead on hardware transactions, which as we prove is inherent to such HyTM designs (Theorem 9). While these HyTM implementations satisfy progressiveness, they do not provide uninstrumented writes. However, the HyTM implementation described in Algorithm 1 is provably opaque, satisfies progressiveness and provides invisible reads. Additionally, it uses uninstrumented writes and is optimal with respect to hardware code instrumentation.

Experiments suggest that the cost of concurrency detection is a significant bottleneck for many HyTM implementations [29], which serves as a major motivation for our definition of instrumentation. Implementations like PhTM [28] and HybridNOrec [9] overcome the per-access instrumentation cost of [11,26] by realizing that if one is prepared to sacrifice progress, hardware transactions need instrumentation only at the boundaries of transactions to detect pending software transactions. Inspired by this observation, our HyTM implementation described in Algorithm 2 overcomes the lower bound of Theorem 9 by allowing hardware readers to abort due to a concurrent software writer, but maintains progressiveness for software transactions, unlike $9,28,29$.

Recent work has investigated alternatives to STM fallback, such as sandboxing [2, 8], and fallback to reduced hardware transactions [29]. These proposals are not currently covered by our framework, although we believe that our model can be extended to incorporate such techniques.

Detailed coverage on HyTM implementations and integration with HTM proposals can be found in 21. An overview of popular HyTM designs and a comparison of the TM properties and instrumentation overhead they incur may be found in 32 .

\section{Concluding remarks}

We have introduced an analytical model for hybrid transactional memory that captures the notion of cached accesses as performed by hardware transactions. We then derived lower and upper bounds in this model to capture the inherent tradeoff between the degree of concurrency allowed between hardware and software transactions and the instrumentation overhead introduced on the hardware. In a nutshell, our results say that it is impossible to completely forgo 
instrumentation in a sequentially consistent HyTM, and that any opaque HyTM implementation providing non-trivial progress either has to pay a linear number of metadata accesses, or will have to allow slow-path transactions to abort fast-path operations.

Several papers have recently proposed the use of both direct and cached accesses within the same transaction to reduce the instrumentation overhead $[26,32,33$, although, to the best of our knowledge, no industrial HTM currently supports this functionality. Another recent approach proposed reduced hardware transactions [29], where part of the slow-path is executed using a short hardware transaction, which allows to eliminate part of the instrumentation from the hardware fast-path. We believe that our model can be extended to incorporate such schemes as well, and we conjecture that the lower bounds established in Theorems 4 and 9 would also hold in the extended model. Future work also includes deriving lower bounds for HyTMs satisfying wider criteria of consistency and progress, and exploring other complexity metrics. 


\section{References}

[1] Advanced Synchronization Facility Proposed Architectural Specification, March 2009. http://developer.amd.com/wordpress/media/2013/09/45432-ASF_Spec_2.1.pdf.

[2] Y. Afek, A. Levy, and A. Morrison. Software-improved hardware lock elision. In PODC. ACM, 2014.

[3] D. Alistarh, P. Eugster, M. Herlihy, A. Matveev, and N. Shavit. Stacktrack: An automated transactional approach to concurrent memory reclamation. In Proceedings of the Ninth European Conference on Computer Systems, EuroSys '14, pages 25:1-25:14, New York, NY, USA, 2014. ACM.

[4] C. S. Ananian, K. Asanovic, B. C. Kuszmaul, C. E. Leiserson, and S. Lie. Unbounded transactional memory. In Proceedings of the 11th International Symposium on High-Performance Computer Architecture, HPCA '05, pages 316-327, Washington, DC, USA, 2005. IEEE Computer Society.

[5] H. Attiya, S. Hans, P. Kuznetsov, and S. Ravi. Safety of deferred update in transactional memory. 2013 IEEE 33rd International Conference on Distributed Computing Systems, 0:601-610, 2013.

[6] H. Attiya and E. Hillel. The cost of privatization in software transactional memory. IEEE Trans. Computers, 62(12):2531-2543, 2013.

[7] H. Attiya, E. Hillel, and A. Milani. Inherent limitations on disjoint-access parallel implementations of transactional memory. Theory of Computing Systems, 49(4):698-719, 2011.

[8] I. Calciu, T. Shpeisman, G. Pokam, and M. Herlihy. Improved single global lock fallback for best-effort hardware transactional memory. In Transact 2014 Workshop. ACM, 2014.

[9] L. Dalessandro, F. Carouge, S. White, Y. Lev, M. Moir, M. L. Scott, and M. F. Spear. Hybrid NOrec: a case study in the effectiveness of best effort hardware transactional memory. In R. Gupta and T. C. Mowry, editors, ASPLOS, pages 39-52. ACM, 2011.

[10] L. Dalessandro, M. F. Spear, and M. L. Scott. Norec: Streamlining stm by abolishing ownership records. SIGPLAN Not., 45(5):67-78, Jan. 2010.

[11] P. Damron, A. Fedorova, Y. Lev, V. Luchangco, M. Moir, and D. Nussbaum. Hybrid transactional memory. SIGPLAN Not., 41(11):336-346, Oct. 2006.

[12] D. Dice, Y. Lev, M. Moir, and D. Nussbaum. Early experience with a commercial hardware transactional memory implementation. In Proceedings of the 14th International Conference on Architectural Support for Programming Languages and Operating Systems, ASPLOS XIV, pages 157-168, New York, NY, USA, 2009. ACM.

[13] D. Dice, O. Shalev, and N. Shavit. Transactional locking ii. In Proceedings of the 20th International Conference on Distributed Computing, DISC'06, pages 194-208, Berlin, Heidelberg, 2006. Springer-Verlag.

[14] A. Dragojević, M. Herlihy, Y. Lev, and M. Moir. On the power of hardware transactional memory to simplify memory management. In Proceedings of the 30th Annual ACM SIGACT-SIGOPS Symposium on Principles of Distributed Computing, PODC '11, pages 99-108, New York, NY, USA, 2011. ACM.

[15] F. Ellen, D. Hendler, and N. Shavit. On the inherent sequentiality of concurrent objects. SIAM J. Comput., 41(3):519-536, 2012. 
[16] R. Guerraoui and M. Kapalka. On obstruction-free transactions. In Proceedings of the twentieth annual symposium on Parallelism in algorithms and architectures, SPAA '08, pages 304-313, New York, NY, USA, 2008. ACM.

[17] R. Guerraoui and M. Kapalka. On the correctness of transactional memory. In Proceedings of the 13th ACM SIGPLAN Symposium on Principles and Practice of Parallel Programming, PPoPP '08, pages 175-184, New York, NY, USA, 2008. ACM.

[18] R. Guerraoui and M. Kapalka. The semantics of progress in lock-based transactional memory. SIGPLAN Not., 44(1):404-415, Jan. 2009.

[19] R. Guerraoui and M. Kapalka. Transactional memory: Glimmer of a theory. In Proceedings of the 21st International Conference on Computer Aided Verification, CAV '09, pages 1-15, Berlin, Heidelberg, 2009. Springer-Verlag.

[20] R. Guerraoui and M. Kapalka. Principles of Transactional Memory,Synthesis Lectures on Distributed Computing Theory. Morgan and Claypool, 2010.

[21] T. Harris, J. R. Larus, and R. Rajwar. Transactional Memory, 2nd edition. Synthesis Lectures on Computer Architecture. Morgan \& Claypool Publishers, 2010.

[22] M. Herlihy. Wait-free synchronization. ACM Trans. Prog. Lang. Syst., 13(1):123-149, 1991.

[23] M. Herlihy, V. Luchangco, M. Moir, and W. N. Scherer, III. Software transactional memory for dynamic-sized data structures. In Proceedings of the Twenty-second Annual Symposium on Principles of Distributed Computing, PODC '03, pages 92-101, New York, NY, USA, 2003. ACM.

[24] M. Herlihy and J. E. B. Moss. Transactional memory: architectural support for lock-free data structures. In ISCA, pages 289-300, 1993.

[25] A. Israeli and L. Rappoport. Disjoint-access-parallel implementations of strong shared memory primitives. In $P O D C$, pages 151-160, 1994.

[26] S. Kumar, M. Chu, C. J. Hughes, P. Kundu, and A. Nguyen. Hybrid transactional memory. In Proceedings of the Eleventh ACM SIGPLAN Symposium on Principles and Practice of Parallel Programming, PPoPP '06, pages 209-220, New York, NY, USA, 2006. ACM.

[27] P. Kuznetsov and S. Ravi. On the cost of concurrency in transactional memory. CoRR, abs/1103.1302, 2011.

[28] Y. Lev, M. Moir, and D. Nussbaum. Phtm: Phased transactional memory. In In Workshop on Transactional Computing (Transact), 200\%. research.sun.com/scalable/pubs/ TRANSACT2007PhTM.pdf.

[29] A. Matveev and N. Shavit. Reduced hardware transactions: a new approach to hybrid transactional memory. In Proceedings of the 25th ACM symposium on Parallelism in algorithms and architectures, pages 11-22. ACM, 2013.

[30] M. Ohmacht. Memory Speculation of the Blue Gene/Q Compute Chip, 2011. http: //wands.cse.lehigh.edu/IBM_BQC_PACT2011.ppt.

[31] J. Reinders. Transactional Synchronization in Haswell, 2012. http://software.intel. com/en-us/blogs/2012/02/07/transactional-synchronization-in-haswell/.

[32] T. Riegel. Software Transactional Memory Building Blocks. 2013. 
[33] T. Riegel, P. Marlier, M. Nowack, P. Felber, and C. Fetzer. Optimizing hybrid transactional memory: The importance of nonspeculative operations. In Proceedings of the 23rd ACM Symposium on Parallelism in Algorithms and Architectures, pages 53-64. ACM, 2011.

\section{A Progressive opaque HyTM implementation that provides unin- strumented writes and invisible reads}

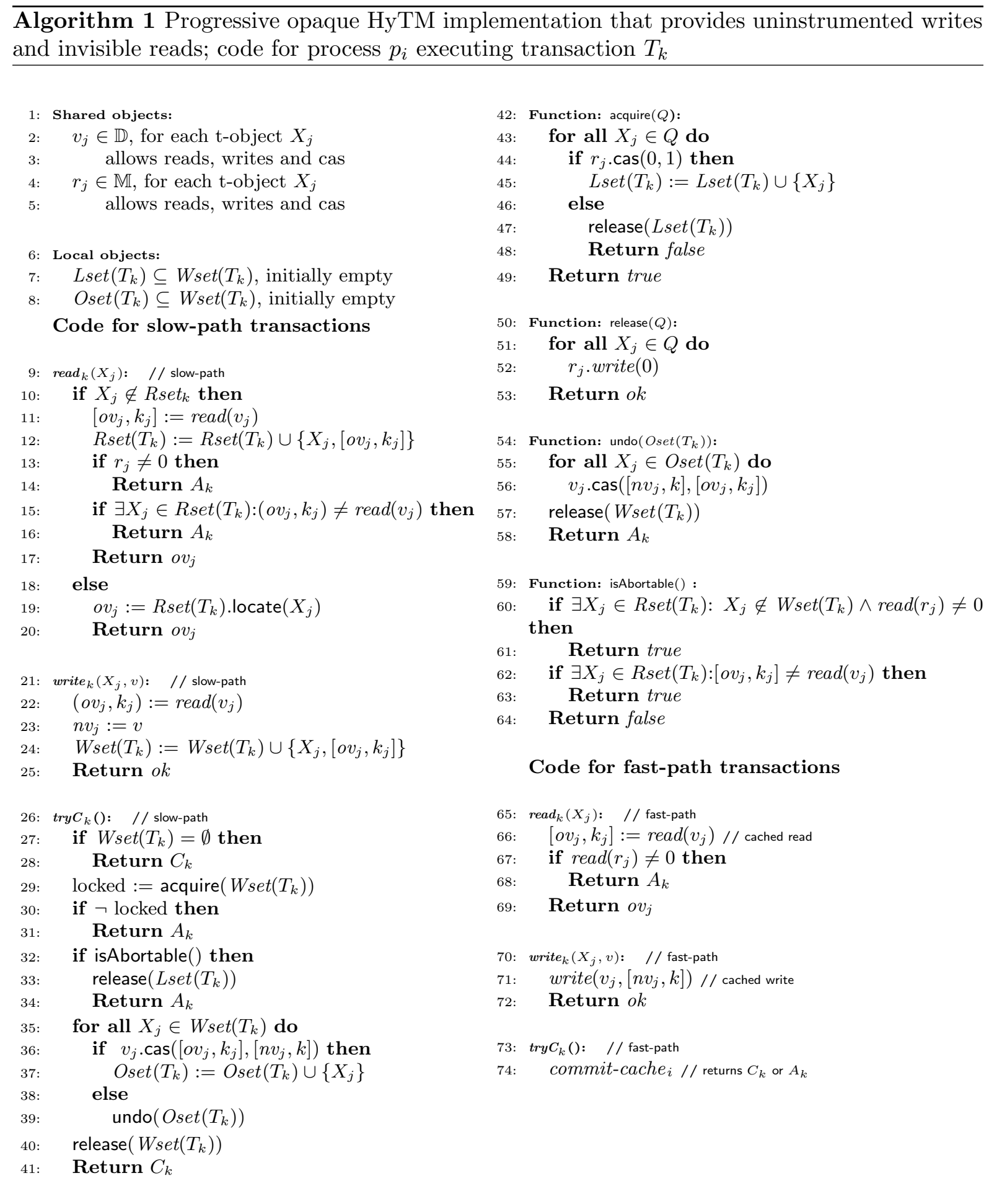

Let $E$ be a t-sequential execution. For every operation $\operatorname{read}_{k}(X)$ in $E$, we define the latest 
written value of $X$ as follows: (1) If $T_{k}$ contains a write $_{k}(X, v)$ preceding $\operatorname{read}_{k}(X)$, then the latest written value of $X$ is the value of the latest such write to $X$. (2) Otherwise, if $E$ contains a write $_{m}(X, v), T_{m}$ precedes $T_{k}$, and $T_{m}$ commits in $E$, then the latest written value of $X$ is the value of the latest such write to $X$ in $E$. (This write is well-defined since $E$ starts with $T_{0}$ writing to all t-objects.) We say that $\operatorname{read}_{k}(X)$ is legal in a t-sequential execution $E$ if it returns the latest written value of $X$, and $E$ is legal if every $\operatorname{read}_{k}(X)$ in $H$ that does not return $A_{k}$ is legal in $E$.

For a history $H$, a completion of $H$, denoted $\bar{H}$, is a history derived from $H$ as follows:

1. for every incomplete t-operation $o p_{k}$ that is a read $\vee \vee$ write $e_{k}$ of $T_{k} \in \operatorname{txns}(H)$ in $H$, insert $A_{k}$ somewhere after the last event of $T_{k}$ in $E$; otherwise if $o p_{k}=\operatorname{try} C_{k}$, insert $A_{k}$ or $C_{k}$ somewhere after the last event of $T_{k}$

2. for every complete transaction $T_{k}$ in the history derived in (1) that is not t-complete, insert $\operatorname{try} C_{k} \cdot A_{k}$ after the last event of transaction $T_{k}$.

Definition 6 (Opacity and strict serializability). A finite history $H$ is opaque if there is a legal $t$-complete t-sequential history $S$, such that for any two transactions $T_{k}, T_{m} \in \operatorname{txns}(H)$, if $T_{k}$ precedes $T_{m}$ in real-time order, then $T_{k}$ precedes $T_{m}$ in $S$, and $S$ is equivalent to a completion of $H$ [20].

A finite history $H$ is strictly serializable if there is a legal $t$-complete $t$-sequential history $S$, such that for any two transactions $T_{k}, T_{m} \in \operatorname{txns}(H)$, if $T_{k} \prec_{H}^{R T} T_{m}$, then $T_{k}$ precedes $T_{m}$ in $S$, and $S$ is equivalent to $\operatorname{cseq}(\bar{H})$, where $\bar{H}$ is some completion of $H$ and cseq $(\bar{H})$ is the subsequence of $\bar{H}$ reduced to committed transactions in $\bar{H}$.

We refer to $S$ as a serialization of $H$.

Lemma 15. Algorithm 1 implements an opaque TM.

Proof. Let $E$ by any execution of Algorithm 1. Since opacity is a safety property, it is sufficient to prove that every finite execution is opaque [5]. Let $<_{E}$ denote a total-order on events in $E$.

Let $H$ denote a subsequence of $E$ constructed by selecting linearization points of t-operations performed in $E$. The linearization point of a t-operation $o p$, denoted as $\ell_{o p}$ is associated with a base object event or an event performed during the execution of op using the following procedure.

Completions. First, we obtain a completion of $E$ by removing some pending invocations or adding responses to the remaining pending invocations as follows:

- incomplete read ${ }_{k}$, write $e_{k}$ operation performed by a slow-path transaction $T_{k}$ is removed from $E$; an incomplete try $C_{k}$ is removed from $E$ if $T_{k}$ has not performed any write to a base object $r_{j} ; X_{j} \in W \operatorname{set}\left(T_{k}\right)$ in Line 36, otherwise it is completed by including $C_{k}$ after E.

- every incomplete read $_{k}, \operatorname{try} A_{k}$, write $e_{k}$ and $\operatorname{try} C_{k}$ performed by a fast-path transaction $T_{k}$ is removed from $E$.

Linearization points. Now a linearization $H$ of $E$ is obtained by associating linearization points to t-operations in the obtained completion of $E$. For all t-operations performed a slowpath transaction $T_{k}$, linearization points as assigned as follows:

- For every t-read $o p_{k}$ that returns a non- $\mathrm{A}_{k}$ value, $\ell_{o p_{k}}$ is chosen as the event in Line 11 of Algorithm 1, else, $\ell_{o p_{k}}$ is chosen as invocation event of $o p_{k}$

- For every $o p_{k}=$ write $_{k}$ that returns, $\ell_{o p_{k}}$ is chosen as the invocation event of $o p_{k}$

- For every $o p_{k}=\operatorname{try} C_{k}$ that returns $C_{k}$ such that $W \operatorname{set}\left(T_{k}\right) \neq \emptyset, \ell_{o p_{k}}$ is associated with the first write to a base object performed by release when invoked in Line 40, else if $o p_{k}$ returns $A_{k}, \ell_{o p_{k}}$ is associated with the invocation event of $o p_{k}$

- For every $o p_{k}=\operatorname{try} C_{k}$ that returns $C_{k}$ such that $W \operatorname{set}\left(T_{k}\right)=\emptyset, \ell_{o p_{k}}$ is associated with Line 28

For all t-operations performed a fast-path transaction $T_{k}$, linearization points as assigned as follows:

- For every t-read $o p_{k}$ that returns a non- $\mathrm{A}_{k}$ value, $\ell_{o p_{k}}$ is chosen as the event in Line 66 of Algorithm 1, else, $\ell_{o p_{k}}$ is chosen as invocation event of $o p_{k}$ 
- For every $o p_{k}$ that is a $\operatorname{try} C_{k}, \ell_{o p_{k}}$ is the commit-cache $e_{k}$ primitive invoked by $T_{k}$

- For every $o p_{k}$ that is a write $e_{k}, \ell_{o p_{k}}$ is the event in Line 71 . $<_{H}$ denotes a total-order on t-operations in the complete sequential history $H$.

Serialization points. The serialization of a transaction $T_{j}$, denoted as $\delta_{T_{j}}$ is associated with the linearization point of a t-operation performed by the transaction.

We obtain a t-complete history $\bar{H}$ from $H$ as follows. A serialization $S$ is obtained by associating serialization points to transactions in $\bar{H}$ as follows: for every transaction $T_{k}$ in $H$ that is complete, but not t-complete, we insert $\operatorname{try} C_{k} \cdot A_{k}$ immediately after the last event of $T_{k}$ in $H$.

- If $T_{k}$ is an updating transaction that commits, then $\delta_{T_{k}}$ is $\ell_{t r y} C_{k}$

- If $T_{k}$ is a read-only or aborted transaction, then $\delta_{T_{k}}$ is assigned to the linearization point of the last t-read that returned a non- $\mathrm{A}_{k}$ value in $T_{k}$

$<_{S}$ denotes a total-order on transactions in the t-sequential history $S$.

Claim 16. If $T_{i} \prec_{H} T_{j}$, then $T_{i}<_{S} T_{j}$

Proof. This follows from the fact that for a given transaction, its serialization point is chosen between the first and last event of the transaction implying if $T_{i} \prec_{H} T_{j}$, then $\delta_{T_{i}}<_{E} \delta_{T_{j}}$ implies $T_{i}<_{S} T_{j}$.

Claim 17. $S$ is legal.

Proof. We claim that for every $\operatorname{read}_{j}\left(X_{m}\right) \rightarrow v$, there exists some slow-path transaction $T_{i}$ (or resp. fast-path) that performs write $\left(X_{m}, v\right)$ and completes the event in Line 36 (or resp. Line 71) such that $\operatorname{read}_{j}\left(X_{m}\right) \nprec_{H}^{R T}$ write $_{i}\left(X_{m}, v\right)$.

Suppose that $T_{i}$ is a slow-path transaction: since $\operatorname{read}_{j}\left(X_{m}\right)$ returns the response $v$, the event in Line 11 succeeds the event in Line 36 performed by $\operatorname{try} C_{i}$. Since $\operatorname{read}_{j}\left(X_{m}\right)$ can return a nonabort response only after $T_{i}$ writes 0 to $r_{m}$ in Line $52, T_{i}$ must be committed in $S$. Consequently, $\ell_{\text {try } C_{i}}<_{E} \ell_{\text {read }_{j}\left(X_{m}\right)}$. Since, for any updating committing transaction $T_{i}, \delta_{T_{i}}=\ell_{\text {try }} C_{i}$, it follows that $\delta_{T_{i}}<_{E} \delta_{T_{j}}$.

Otherwise if $T_{i}$ is a fast-path transaction, then clearly $T_{i}$ is a committed transaction in $S$. Recall that $\operatorname{read}_{j}\left(X_{m}\right)$ can read $v$ during the event in Line 11 only after $T_{i}$ applies the commit-cache primitive. By the assignment of linearization points, $\ell_{t r y C_{i}}<_{E} \ell_{\text {read }_{j}\left(X_{m}\right)}$ and thus, $\delta_{T_{i}}<_{E} \ell_{\text {read }_{j}\left(X_{m}\right)}$.

Thus, to prove that $S$ is legal, it suffices to show that there does not exist a transaction $T_{k}$ that returns $C_{k}$ in $S$ and performs write $e_{k}\left(X_{m}, v^{\prime}\right) ; v^{\prime} \neq v$ such that $T_{i}<_{S} T_{k}<_{S} T_{j}$.

$T_{i}$ and $T_{k}$ are both updating transactions that commit. Thus,

$$
\begin{gathered}
\left(T_{i}<_{S} T_{k}\right) \Longleftrightarrow\left(\delta_{T_{i}}<_{E} \delta_{T_{k}}\right) \\
\left(\delta_{T_{i}}<_{E} \delta_{T_{k}}\right) \Longleftrightarrow\left(\ell_{\text {try } C_{i}}<_{E} \ell_{\text {try }}\right)
\end{gathered}
$$

Since, $T_{j}$ reads the value of $X$ written by $T_{i}$, one of the following is true: $\ell_{t r y C_{i}}<_{E} \ell_{t r y C_{k}}<_{E}$ $\ell_{\text {read }}\left(X_{m}\right)$ or $\ell_{\text {try } C_{i}}<_{E} \ell_{\text {read }_{j}\left(X_{m}\right)}<_{E} \ell_{\text {try }} C_{k}$.

Suppose that $\ell_{\text {try } C_{i}}<_{E} \ell_{\text {try }} C_{k}<_{E} \ell_{\text {read }}\left(X_{m}\right)$.

(Case I:) $T_{i}$ and $T_{k}$ are slow-path transactions.

Thus, $T_{k}$ returns a response from the event in Line 29 before the read of the base object associated with $X_{m}$ by $T_{j}$ in Line 11 . Since $T_{i}$ and $T_{k}$ are both committed in $E, T_{k}$ returns true from the event in Line 29 only after $T_{i}$ writes 0 to $r_{m}$ in Line 52 .

If $T_{j}$ is a slow-path transaction, recall that $\operatorname{read}_{j}\left(X_{m}\right)$ checks if $X_{j}$ is locked by a concurrent transaction, then performs read-validation (Line 13) before returning a matching response. We claim that $\operatorname{read}_{j}\left(X_{m}\right)$ must return $A_{j}$ in any such execution.

Consider the following possible sequence of events: $T_{k}$ returns true from acquire function invocation, updates the value of $X_{m}$ to shared-memory (Line 36), $T_{j}$ reads the base object $v_{m}$ associated with $X_{m}, T_{k}$ releases $X_{m}$ by writing 0 to $r_{m}$ and finally $T_{j}$ performs the check in Line 13. But in this case, $\operatorname{read}_{j}\left(X_{m}\right)$ is forced to return the value $v^{\prime}$ written by $T_{m}$ contradiction to the assumption that $\operatorname{read}_{j}\left(X_{m}\right)$ returns $v$. 
Otherwise suppose that $T_{k}$ acquires exclusive access to $X_{m}$ by writing 1 to $r_{m}$ and returns true from the invocation of acquire, updates $v_{m}$ in Line 36), $T_{j}$ reads $v_{m}, T_{j}$ performs the check in Line 13 and finally $T_{k}$ releases $X_{m}$ by writing 0 to $r_{m}$. Again, $\operatorname{read}_{j}\left(X_{m}\right)$ must return $A_{j}$ since $T_{j}$ reads that $r_{m}$ is 1 - contradiction.

A similar argument applies to the case that $T_{j}$ is a fast-path transaction. Indeed, since every data base object read by $T_{j}$ is contained in its tracking set, if any concurrent transaction updates any t-object in its read set, $T_{j}$ is aborted immediately by our model(cf. Section 3).

Thus, $\ell_{\text {try }} C_{i}<_{E} \ell_{\text {read }_{j}(X)}<_{E} \ell_{\text {try }} C_{k}$.

(Case II:) $T_{i}$ is a slow-path transaction and $T_{k}$ is a fast-path transaction. Thus, $T_{k}$ returns $C_{k}$ before the read of the base object associated with $X_{m}$ by $T_{j}$ in Line 11, but after the response of acquire by $T_{i}$ in Line 29. Since $\operatorname{read}_{j}\left(X_{m}\right)$ reads the value of $X_{m}$ to be $v$ and not $v^{\prime}$, $T_{i}$ performs the cas to $v_{m}$ in Line 36 after the $T_{k}$ performs the commit-cache primitive (since if otherwise, $T_{k}$ would be aborted in $E$ ). But then the cas on $v_{m}$ performed by $T_{i}$ would return false and $T_{i}$ would return $A_{i}$-contradiction.

(Case III:) $T_{k}$ is a slow-path transaction and $T_{i}$ is a fast-path transaction. This is analogous to the above case.

(Case $I V:$ :) $T_{i}$ and $T_{k}$ are fast-path transactions. Thus, $T_{k}$ returns $C_{k}$ before the read of the base object associated with $X_{m}$ by $T_{j}$ in Line 11, but before $T_{i}$ returns $C_{i}$ (this follows from Observations 1 and 22. Consequently, read $_{j}\left(X_{m}\right)$ must read the value of $X_{m}$ to be $v^{\prime}$ and return $v^{\prime}$-contradiction.

We now need to prove that $\delta_{T_{j}}$ indeed precedes $\ell_{t r y C_{k}}$ in $E$.

Consider the two possible cases:

- Suppose that $T_{j}$ is a read-only transaction. Then, $\delta_{T_{j}}$ is assigned to the last t-read performed by $T_{j}$ that returns a non- $\mathrm{A}_{j}$ value. If $\operatorname{read}_{j}\left(X_{m}\right)$ is not the last t-read that returned a non- $\mathrm{A}_{j}$ value, then there exists a $\operatorname{read}_{j}\left(X^{\prime}\right)$ such that $\ell_{\text {read }_{j}\left(X_{m}\right)}<_{E} \ell_{t r y C_{k}}<_{E}$ $\ell_{\text {read }_{j}\left(X^{\prime}\right)}$. But then this t-read of $X^{\prime}$ must abort by performing the checks in Line 13 or incur a tracking set abort-contradiction.

- Suppose that $T_{j}$ is an updating transaction that commits, then $\delta_{T_{j}}=\ell_{t r y C_{j}}$ which implies that $\ell_{\text {read }}(X)<_{E} \ell_{t r y C_{k}}<_{E} \ell_{t r y C_{j}}$. Then, $T_{j}$ must neccesarily perform the checks in Line 32 and return $A_{j}$ or incur a tracking set abort-contradiction to the assumption that $T_{j}$ is a committed transaction.

The proof follows.

The conjunction of Claims 16 and 17 establish that Algorithm 1 is opaque.

Theorem 18 (Theorem 13). There exists an opaque HyTM implementation $\mathcal{M}$ that provides uninstrumented writes, invisible reads, progressiveness and wait-free TM-liveness such that in every execution $E$ of $\mathcal{M}$, every read-only fast-path transaction $T \in$ txns $(E)$ accesses $O(|R \operatorname{Ret}(T)|)$ distinct metadata base objects.

Proof. (TM-liveness and TM-progress) Since none of the implementations of the t-operations in Algorithm 1 contain unbounded loops or waiting statements, Algorithm 1 provides wait-free TM-liveness i.e. every t-operation returns a matching response after taking a finite number of steps.

Consider the cases under which a slow-path transaction $T_{k}$ may be aborted in any execution.

- Suppose that there exists a $\operatorname{read}_{k}\left(X_{j}\right)$ performed by $T_{k}$ that returns $A_{k}$ from Line 13. Thus, there exists a transaction that has written 1 to $r_{j}$ in Line 44 , but has not yet written 0 to $r_{j}$ in Line 52 or some t-object in $\operatorname{Rset}\left(T_{k}\right)$ has been updated since its t-read by $T_{k}$. In both cases, there exists a concurrent transaction performing a t-write to some t-object in $R \operatorname{set}\left(T_{k}\right)$, thus forcing a read-write conflict.

- Suppose that $\operatorname{try} C_{k}$ performed by $T_{k}$ that returns $A_{k}$ from Line 30. Thus, there exists a transaction that has written 1 to $r_{j}$ in Line 44, but has not yet written 0 to $r_{j}$ in Line 52 . Thus, $T_{k}$ encounters write-write conflict with another transaction that concurrently attempts to update a t-object in $W \operatorname{set}\left(T_{k}\right)$. 
- Suppose that $\operatorname{try} C_{k}$ performed by $T_{k}$ that returns $A_{k}$ from Line 32 . Since $T_{k}$ returns $A_{k}$ from Line 32 for the same reason it returns $A_{k}$ after Line 13 , the proof follows.

Consider the cases under which a fast-path transaction $T_{k}$ may be aborted in any execution $E$.

- Suppose that a read $_{k}\left(X_{m}\right)$ performed by $T_{k}$ returns $A_{k}$ from Line 67. Thus, there exists a concurrent slow-path transaction that is pending in its tryCommit and has written 1 to $r_{m}$, but not released the lock on $X_{m}$ i.e. $T_{k}$ conflicts with another transaction in $E$.

- Suppose that $T_{k}$ returns $A_{k}$ while performing a cached access of some base object $b$ via a trivial (and resp. nontrivial) primitive. Indeed, this is possible only if some concurrent transaction writes (and resp. reads or writes) to $b$. However, two transactions $T_{k}$ and $T_{m}$ may contend on $b$ in $E$ only if there exists $X \in \operatorname{Dset}\left(T_{i}\right) \cap \operatorname{Dset}\left(T_{j}\right)$ and $X \in W \operatorname{set}\left(T_{i}\right) \cup$ $W \operatorname{set}\left(T_{j}\right)$. from Line 30. The same argument applies for the case when $T_{k}$ returns $A_{k}$ while performing commit-cache ${ }_{k}$ in $E$.

(Complexity) The implementation uses uninstrumented writes since each write $_{k}\left(X_{m}\right)$ simply writes to $v_{m} \in \mathbb{D}_{X_{m}}$ and does not access any metadata base object. The complexity of each $\operatorname{read}_{k}\left(X_{m}\right)$ is a single access to a metadata base object $r_{m}$ in Line 67 that is not accessed any other transaction $T_{i}$ unless $X_{m} \in \operatorname{Dset}\left(T_{i}\right)$. while the $\operatorname{try} C_{k}$ just calls cache-commit $k_{k}$ that returns $C_{k}$. Thus, each read-only transaction $T_{k}$ accesses $O\left(\left|R \operatorname{Ret}\left(T_{k}\right)\right|\right)$ distinct metadata base objects in any execution.

\section{B Opaque HyTM implementation with invisible reads that is progressive only for slow-path transactions}

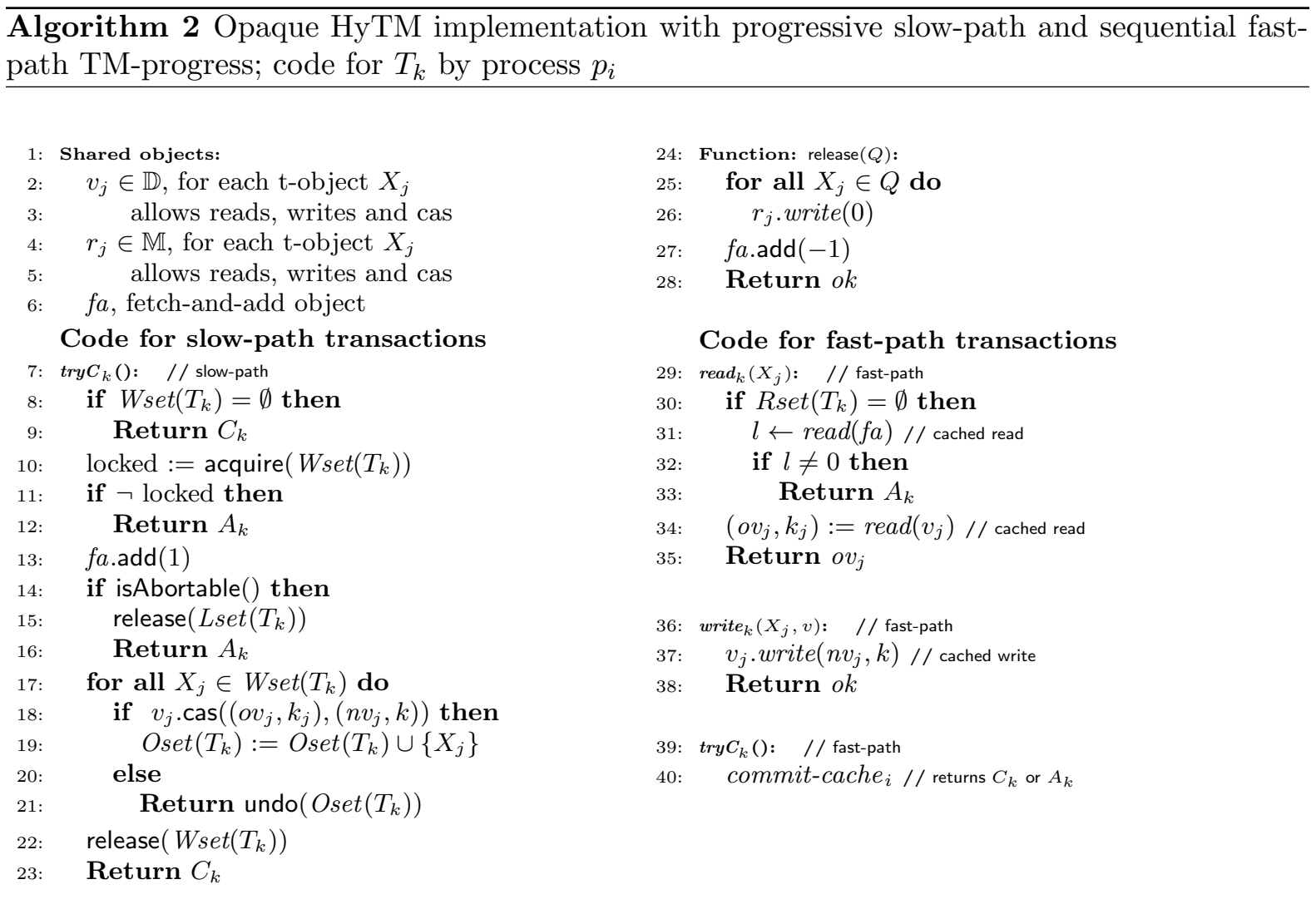

Theorem 19 (Theorem 14). There exists an opaque HyTM implementation $\mathcal{M}$ that provides invisible reads, progressiveness for slow-path transactions, sequential TM-progress for fast-path transactions and wait-free TM-liveness such that in every execution $E$ of $\mathcal{M}$, every fast-path transaction accesses at most one metadata base object. 
Proof. The proof of opacity is almost identical to the analogous proof for Algorithm 1 in Lemma 15.

As with Algorithm 1, enumerating the cases under which a slow-path transaction $T_{k}$ returns $A_{k}$ proves that Algorithm 2 satisfies progressiveness for slow-path transactions. Any fast-path transaction $T_{k} ; \operatorname{Rset}\left(T_{k}\right) \neq \emptyset$ reads the metadata base object $f a$ and adds it to the process's tracking set (Line 31). If the value of $f a$ is not 0 , indicating that there exists a concurrent slowpath transaction pending in its tryCommit, $T_{k}$ returns $A_{k}$. Thus, the implementation provides sequential TM-progress for fast-path transactions.

Also, in every execution $E$ of $\mathcal{M}$, no fast-path write-only transaction accesses any metadata base object and a fast-path reading transaction accesses the metadata base object $f a$ exactly once, during the first t-read. 